\title{
Spreading Bets or Going 'All-In'? Navigating Consortia-led Digital Innovation Pathways
}

\author{
Tomás Seosamh Harrington $^{a}$, Nitin R. Joglekar ${ }^{b}$ and Jagjit Singh Srai ${ }^{\mathrm{c}}$ \\ alnnovation, Technology and Operations Management Group, Norwich Business School, University of \\ East Anglia (UEA), Norwich NR4 7TJ, UK. tomas.harrington@uea.ac.uk \\ ${ }^{\mathrm{b}}$ Questrom School of Business, Boston University, Boston MA 02215, USA. joglekar@bu.edu \\ ${ }^{\circ}$ Centre for International Manufacturing, Institute for Manufacturing, University of Cambridge, \\ Cambridge CB3 0FS, UK. jss46@cam.ac.uk
}

\begin{abstract}
This paper explores the unique tensions and practical dynamics of pre-competitive consortia. As consortia can involve supply chain organizations, together with regulatory bodies and universities, participation is predicated by partners with markedly different outcome goals and risk dispositions. Some are willing to go 'all-in' for a long-term vision involving novel breakthroughs; others are focused on specific incremental gains. We draw from an eight-year dataset involving 98 entities, collaborating across five UK-based pharmaceutical sector consortia. We present case evidence from 14 projects where consortia are experimenting with a wide variety of digital initiatives, ranging from incremental to potentially game-changing (novel) innovations. A central issue here is the availability of alternative digital innovation pathways. So which pathway to take?

We follow an abductive approach to examine the nature of these pathways and how consortia are organizing efforts for specific innovation performance and allied risk outcomes. We find that sequential pathways are characterized by 'tactical gains'. Here, 'attractive' cases determine the pathway choice, resulting in follow-on incremental activities. In contrast, simultaneous pathways require hypotheses development where immediate ROls are not always apparent. Here, the consortium effect 'de-risks risk conversations', enabling partners to get internal buy-in. Navigating such pathways within consortia has theory and practice implications. For example, a surprising finding is the going 'all-in' approach, often seen as loaded with risk, can be appropriate from a risk mitigation perspective for novel projects. As is the case with emerging consortia-led Covid-19 vaccines, where steps that are normally taken sequentially are being carried out simultaneously.
\end{abstract}

Keywords: Consortia; Digitalization; Risk Mitigation; Project Management

\section{Introduction}

Advancements in digitalization across multiple technology disciplines has brought new requirements for network-centric innovation (Nambisan et al., 2017) as no single firm has the capability to fully exploit independently. This has led firms to enter pre-competitive collaborations involving consortia-based partnerships. University assessments of consortia- 
based partnerships have been widely described, however, industry perspectives are less well understood (Frølund and Riedel, 2018). In this in-depth study of pre-competitive consortia within the UK Pharmaceutical sector, spanning 2011-2018 and comprising research projects of value in excess of $\$ 100 \mathrm{~m}$, we have observed two types of innovation: those that involve multiple digital technology innovations within conventional research workflows, activities that broadly follow established design rules; and those that require new breakthrough approaches in support of more novel approaches. In this context, we observed pathways where network-centric digital innovations were managed either sequentially ('spreading bets') or simultaneously (going 'all-in'). In this paper, we explore how operational risks are created and mitigated in each of these distinct pathways.

Given the unique, evolutionary nature of our study, and as academic partners where we co-developed a succession of pre-competitive practitioner-inspired programmes, our scientific reasoning is grounded primarily in the post-factum theoretical interpretation of empirical observations. Through our case observations, we explore consortia efforts in realizing a series of digitalization initiatives with a view to offering plausible, conjecturable explanations (Bamberger, 2018) in the context of risk mitigation. With consortia partners now seeking to make sense of substantial follow-on investments, we look to inform future project selection and pathways to development. Given the unique tensions and practical dynamics at play, we outline how pre-competitive consortia might best organize for network-centric digital innovations, i.e., when to adopt a sequential pathway and when the simultaneous pathway is more appropriate.

\section{Bridging consortia and network-centric innovation research}

While there is significant prior work relating to innovation and networks (Powell et al., 1996), to our knowledge there has been no in-depth studies in the context of pre-competitive consortia engaging in digitalization R\&D. Despite recent proliferation, few studies have assessed the operational effectiveness of consortia in reducing costs and risk (Papadaki and Hirsch, 2013). We pose a central question in whether alternative pathways in consortia- 
based innovation lead to alternative risk outcomes. Our eight year study offers first suggestions here. So how might consortia organize for digital innovations? They could 'spread bets' and try to manage a wide portfolio of projects through multiple 'coopetition' arrangements (Gnyawali and Park, 2011; Pathak et al., 2014). However, these may require very different strategies, in refining existing and developing new knowledge with various levels of complexity (Chandrasekaran et al., 2016). So they might choose to manage projects either sequentially or simultaneously depending on novelty (Pich et al., 2002) or choose to 'hedge bets' and do both. But what proportion of resources should be directed to each type of pathway? From a practice perspective, a sequential or simultaneous pathway is often picked based on a business model, but what does this choice do to firms' risk? In transitioning to digitalization, is the faster route riskier and the slower route less so? Yes seems the obvious answer. However, does this depend on whether the ultimate goal of a project is a conventional (conformance) or novel (performance) outcome?

A central issue we tackle here is the availability of alternative digital innovation pathways within pre-competitive consortia. The literature is yet to address such pathways, where consortia activities span digital product-process and supply chain innovations. Given its focus on collaborative initiatives and shift from firm-centric to network-centric innovation, Nambisan and Sawhney's study on orchestration processes (2011) appears a useful starting point in first understanding the nature of pre-competitive consortia. Second, by offering suggestions in constructing more accurate explanations of innovation processes and outcomes in digital contexts, Nambisan et al., (2017) provide a valuable taxonomy. They outline four new theorizing logics (one of which is orchestration) that are likely to be relevant in making sense of digital innovation pathways. In line with Dhanaraj and Parkhe (2006), we argue that orchestration and the network perspective is particularly relevant to our consortia context. However, while the three network mechanisms of leverage, appropriability, and coherence, have been assessing innovation from a strategic perspective (Nambisan and Sawhney, 2011), from an operational perspective open questions arise if alternative pathways will yield different types of outcome risk. We use these three mechanisms as our 
conceptual foundation and examine how their interplay in pre-competitive consortia settings might shape outcome risks and vary across alternative digital innovation pathways.

We define leverage as: how consortia are integrating unique member-specific knowledge, in terms of assets (innovation design) and relationships (network design); appropriability as: how consortia are realizing value in adopting new knowledge; coherence as: how consortia are coordinating and aligning member outputs and network outcomes in response to environmental conditions. Our unit of analysis is defined as a network-centric digital innovation, i.e., as a consortium-led project designed for specific outputs and outcomes, involving constructs and hypotheses, and with data collection and validation phases (Thomke, 2003). We classify network-centric outputs as those tangible standards, parameters and models that result from a consortium-led project (as opposed to a single focal firm supply network activity). For our practitioners, value creation without thinking about risk is not sufficient, as they are concerned with not just technological difficulty but also risk in regulatory and patient contexts. So we assess outputs in terms of both value creation and risk (conventional v. novel; high v. low); we classify network-centric outcomes as the benefits that a digital innovation is designed to deliver. In practitioner language, we assess in terms of network performance. We pose two central research questions: in leveraging and integrating consortia knowledge through appropriability, what are the risk implications of alternative pathways for conventional and novel outcomes (RQ1)? And what is the role of coherence in moderating and mediating the risk in these pathways (RQ2)?

\subsection{How are pre-competitive consortia organizing for network-centric innovation?}

The basis of this research is that no single firm can fully exploit digitalization R\&D independently. This study is about pre-competitive collaborations involving consortia-based partnerships and not about a single focal firm network. The digital innovations we consider are relevant to several focal firms collaborating at the 'network'-level, yet may be competing at the 'firm'-level. We propose three alternative pathways that best capture network-centric digital innovations (see figure 1). Two pathways are sequential: (1) where digital product- 
process innovations (dPPIs) involve the digitalization of product-process first, and the supply chain may be digitalized subsequently; (2) where digital supply chain innovations (dSCls) involve the digitalization of supply chains first, and product-process may be digitalized subsequently. Finally, a simultaneous pathway represents network-centric digital innovations involving both the digitalization of product-process and the supply chain in parallel.

Practical examples of our dPPI projects include: (i) the digital design of molecules (product). With conventional lab-to-market timelines of 12 years, digitalization efforts around 'predictive capabilities' focus on transitions from laborious physical experimentation and testing to rapid target molecule selection (end goal: to make synthesis of any desired molecule as easy as 'dialling a number'). Outcomes here can facilitate increased success rates in identifying elusive molecules (see our case four on p.17), eliminating non-viable ones, with the potential to collapse development timelines by years and not months;

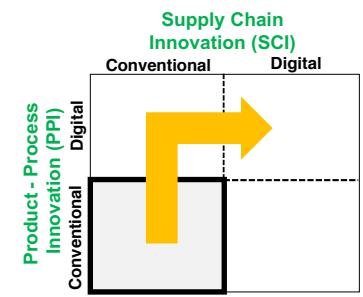

Sequential pathway 1 (dPPI first)

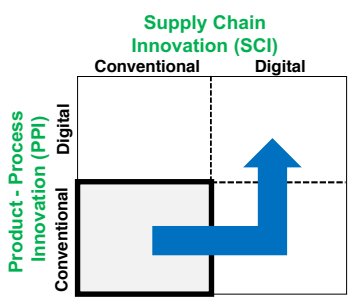

Sequential pathway 2 (dSCl first)

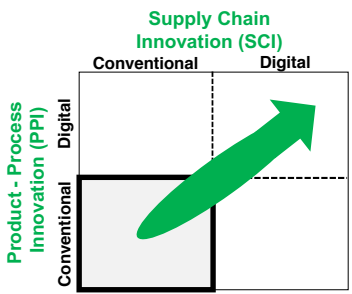
(dPPI and dSCl)

Figure 1. Three alternative digital innovation pathways

(ii) the digital design of routes to manufacture (process). For example, consortia efforts here focus on process analytics, 'real-time' information and 'predictive' control systems for the optimization of complex unit operations. Outcomes can enable the monitoring of critical process variables and their rapid extrapolation from lab to production settings via digital twin development (see our case 11, for an industry-first predictive model, on p.18). Some dSCI projects focus on write-offs of unused clinical stock, which is estimated to be $\$ 10-100$ million for some consortia partners per annum. Practical examples involve new distributed manufacturing supply chain models, often enabled by dPPI outputs, that may offer step changes in volume flexibility and responsiveness. For example, adaptive 'just-in-time' 
technologies reducing lead-times from 4-6 months to $<1$ week (case 8), and process trials with potential savings of $\sim \$ 3$ million on materials and distribution per MNC per year (case 15).

\subsection{Which type of study is appropriate for our context?}

While Nambisan and Sawhney (2011) may provide sufficient basis for formulating our two research questions, it is not possible to derive explicit a priori theoretical hypotheses. As our empirical context and data can ultimately lead to more general theoretical insights, this supports a theory-elaborating approach (Ketokivi and Choi, 2014). We use our base framework (Figure 1) to gather empirical and theoretical insights. To shed new light on potential mechanisms that might enable firms (through their consortia efforts) organize for network-centric digital innovations, we link our observations on pathways and dynamics to mid-range or intermediate theorizing (Edmondson and McManus, 2007). Here, an abductive mode of reasoning can offer first suggestions. Given the nature of our engagement as academic partners in practitioner-led consortia, this is in line with our remit to engage in research that tries to ultimately solve practical problems relating to the implementation of new digital innovations. Also, middle-range theorizing is an appropriate mode of enquiry in developing managerially relevant theories, especially because our application occurs in a specific context. With a limited range of data, this context (alternative digital innovation pathways in consortia setting) becomes of central importance.

The empirical portion of this paper involves a multiple case study design (Yin 2003). The advantage of our design is confirmability and the potential to provide in-depth understanding and appreciation of context. A drawback is lower generalizability and transferability to other contexts. However, we take this 'middle ground' approach as our goal is intermediate insights in a specific consortia context. Our end-goal is not theory building or testing but to offer plausible post-factum explanations. Specifically, ways to make sense of our observed patterns in practice. Our case study approach relies on abductive inference in that we are elaborating on extant theories. Hence, our generalizations and explanations should be 
interpreted as theoretical propositions. The main theory under elaboration is that of orchestration processes in network-centric innovation (Nambisan and Sawhney, 2011).

\section{Research Design}

In this section, we first outline the context behind our industry study. We then summarize our data collection activities between 2011 and 2018, in terms of case selection, consortia engagements, and network analysis. Further details on research phases, data sources, data collection instruments and analytical tools are provided in on-line appendix A1.

\subsection{Industry context and the rise of consortia}

The pharmaceutical sector has a long-standing reputation of being risk averse which has stifled innovation uptake compared to other related industries (Munos, 2009; Harrington et al., 2017). Two common issues that firms face are (i) the cost of poor quality relating to 'batch-to-batch' variability in their processes and (ii) high levels of inventory. An inability to manufacture 'right-first-time' equates to global sector losses of $\$ 20$ billion annually, with firms redirecting $15-20 \%$ of revenues to rework, inspection, and testing (Srai et al., 2015). The value of inventory, for the top 25 MNCs combined, is c. $\$ 100-150$ billion (Daly et al., 2015). These two issues have driven requirements for digitalization and subsequent industry-wide efforts in transforming outmoded practices and performance associated with the traditional 'batch' development-production-testing-'make-to-stock' regime (Leclerc and Smith, 2018). Early consortia efforts (c. 2011) involved predictive analytics and modelling in drug development (Dial-a-Molecule Grand Challenge Network) and the implementation of process analytics and 'continuous' technologies in drug production (CMAC Centre for Innovative Manufacturing). With some early 'wins', consortia members recognized they could capitalize on a wider variety of digitalization opportunities relevant to their respective segments through more pre-competitive collaborations. This culminated in the $\$ 30$ million ReMediES Programme in 2014, with activities designed around supply chain innovation, increasing both network membership and scope. Combined CMAC and ReMediES efforts saw the launch of the Future CMAC Manufacturing Research Hub in 2017, which now 
involves 10 of the largest MNCs (including AstraZeneca; Bayer; GSK; Pfizer) and specialist SMEs working on core technologies. By 2019, the CMAC research portfolio alone comprised of 80 projects, with 18 digital assets showcased. Figure 2 summarizes the make-up of three consortia in our study, some interconnected relationships, and the increasing scale of investment (i.e., $\$ 35$ million UK government funding, matched by $\$ 65$ million from industry).

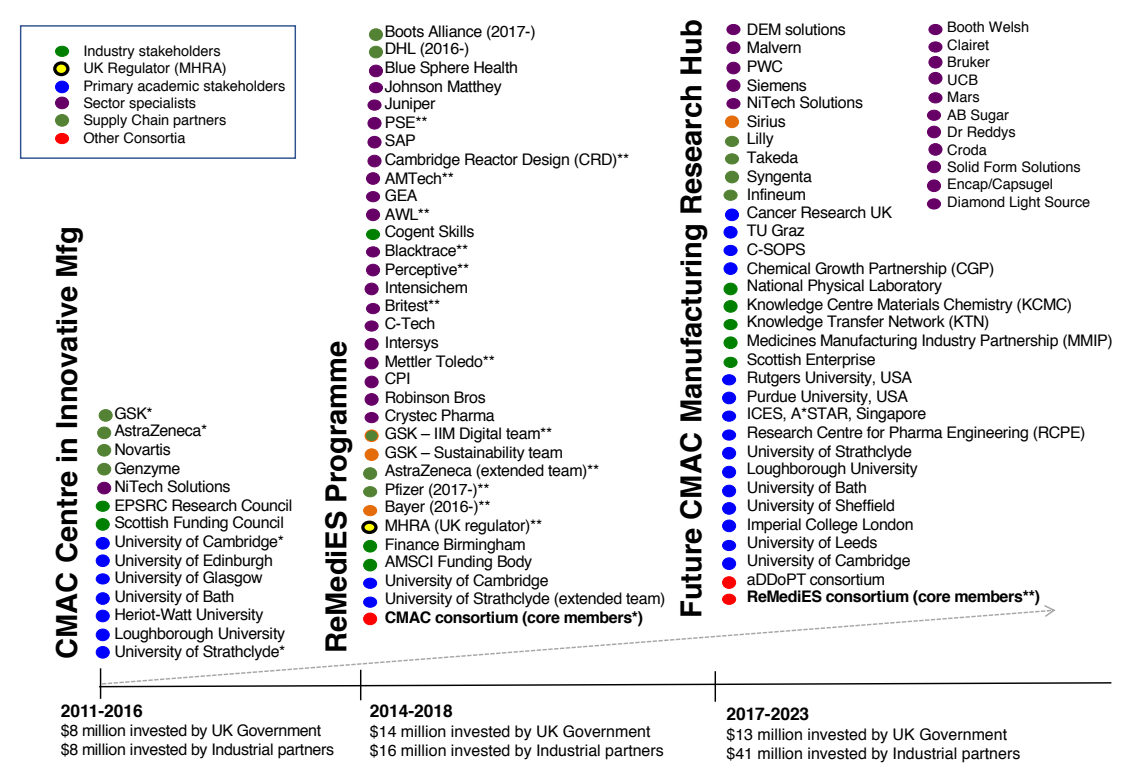

Figure 2. Snapshot example of consortia evolution in terms of members and investment

\subsection{Case Selection}

The first data collection activity involved case selection. With several consortia and literally hundreds of projects to choose from, we first looked to identify a reference population (Yin, 1988). We included in the reference population only entities engaged in pre-competitive consortia that involved $\mathrm{dPPI}$ and $\mathrm{dSCl}$ activities and were collectively assessing new business models in different development-launch-supply scenarios. Given our unit of analysis and sector of interest, our reference population was also constrained to digital innovations involving drug development, production and/or supply. As such, we only considered dPPIs that demonstrated new functionality linked to digitally-enabled testing, validation or production (novel processing equipment and/or analytics), and dSCls with the potential to support new supply models ('make-to-order'). 
We used a combination of industry study approaches (Joglekar et al., 2016; Srai et al., 2016) to construct a current state view of the UK pharmaceutical sector, in order to identify information-rich cases. This served as the basis for capturing key actors, activities (i.e., evidenced dPPIs and dSCls), consortia linkages, and our criterion sampling approach. From this, specific cases were identified from five pre-competitive collaborative UK-based consortia (see Appendix 1) where we evidenced firms accessing resources and capabilities across multiple partners to achieve necessary scale for digitalization R\&D. In order to make inferences and maximise variation across our cases, stratified purposeful sampling (Patton, 1990) was also employed based on specific applications ('off-line' v. 'on-line' testing; batch v. continuous processing; high/low volume and low/high variety contexts). We also looked to strategically arrange our sample into case pairs (where possible) to enable us to tease out more generalizable propositions and theoretical insights (Eisenhardt, 1989).

In summary, 14 network-centric digital innovations were selected for analysis (two additional base cases served as our 'points of departure'). Further details on our sample are outlined as part of our analysis in section 4 (see also Appendices 2-4) and a summary sampling grid (see on-line appendix A2). Cases 2-4 were representative of sequential pathway 1 and involved initiatives around molecule synthesis and crystallization unit operations. These two critical steps occur right at the start of the manufacturing process and determine the purity and particle size of most molecules. In turn, these two quality attributes directly impact other downstream processes and the therapeutic efficacy of overall drug performance. Hence, these specific unit operations are a strategic area of focus in the UK (\$55 million invested in the Future CMAC Manufacturing Hub). Cases 6-8 represented sequential pathway 2 and involved 'intelligent' pack technologies in both commercial and clinical contexts. In line with recent regulations around serialization (FDA, 2019), dSCls focus on the 'track and trace' of drugs in commercial supply chains from point of manufacture to dispense. In a clinical supply context, we focused on how dSCls might enable late postponement and product customization initiatives to eliminate costly inventory write-offs. This is a key sector goal as our consortium partners estimated that between $50-$ 
$75 \%$ of clinical trial material is not dispensed, resulting in unnecessary waste. Finally, cases 9-16 were representative of a simultaneous pathway. Using model (generic) molecules and existing standards as benchmarks, the eight network-centric digital innovations selected focused on achieving comparable performance (if not better and novel) involving selected batch and continuous processes and associated supply chain designs.

\subsection{Consortia engagements}

The second data collection activity involved our consortia engagements. We relied primarily on open-ended interviews with key consortia members and workshop-type engagements that focused on specific consortia goals. The overlapping nature of the five consortia (membership, geography, technologies, activities) enabled our exploration of specific and complementary dPPIs and dSCls, which helped reduce both complexity and variation in terms of fit and context. Where applicable, we targeted engagement with universities, MNCs and SMEs involved in three or more of the consortia for data triangulation efficiency (Eisenhardt and Graebner, 2007). Our role as academic partner in three of the consortia also enabled ongoing access to expert informants over an eight-year period, and allowed for refinement and the gathering of additional data. Hence, we adopted a dynamic approach with respect to case analyses during three phases, and interview protocols in line with themes that emerged from interview and workshop activities (see on-line appendix A1). Specifics on workshop activities and respondents have been previously reported (reference removed). Once key themes emerged from initial rounds of interviews and workshops, we enriched the data with secondary data sources while triangulating information from senior industrialists. Our analysis also used basic coding techniques to try and gain further clarity on different contexts within our selected cases that were especially relevant in answering our research questions. We clustered interview and workshop data in order to open code 'conceptually similar events/actions/interactions' (Corbin and Strauss, 1990, p.12) using the language of practitioners (e.g., did an output ultimately deliver 'consistent', 'better', or 'novel' outcomes?). We organized around our three network mechanisms as a basis of observation 
(in terms of observed inputs and outputs), and subsequently looked for patterns involving themes and variables that emerged. In terms of validation, checks and balances were established and evidenced in contributions linked to targeted consortia outputs, e.g., MITCMAC white paper series (Badman and Trout, 2015).

\subsection{Network Analysis}

The third data collection activity involved network analysis (NA) to draw further inferences for our sample. Given the complexities in effectively engaging with 98 entities across 5 consortia, we worked with central nodes to gather data regarding structure and information flows between key partners (maximum number of nodes was fixed at 24). We used a NA approach where patterns and insights relating to specific communities could be identified (Parraguez et al., 2016). Consistent with their approach, we used the two-step cluster analysis algorithm (IBM Corp, 2001) as the basis for our analysis, because our data incorporated directed graphs. That is, these data mapped on to three networks that were representative of the three alternative pathways outlined in Figure 1. See on-line appendix A3 for graphical representations and further details on the three networks.

A series of 'sub-networks' were identified from our NA and formed the basis of our observations in terms of inputs. Specifying precise inputs was an important task here as we wanted to link to specific consortia outputs. We categorised these observed inputs in terms of leverage, appropriability and coherence to operationalize, measure and compare cases. We deduced insights using metrics from NA and qualitatively through interviews with project leads and relevant experts, triangulating data with case anecdotes and review of relevant peer-reviewed publications. We defined leverage ratio as a measure of the direct connection of unique knowledge sources to the central nodes of the network under study. The basis of observation was the 'Integration' sub-network in each pathway. We defined the appropriability ratio as a measure of the uptake of new routines and standards emerging from network-centric digital innovations. The basis of our observation here was the 'Design Rules' sub-network. Finally, we defined coherence ratio as a measure of 'transition to a 
revised platform' (Nambisan and Sawhney, 2011, p.46). Here, we looked at trade-offs and deduced whether digitalization might ease or constrain performance with the 'Transition' sub-network as our basis of observation.

For subsequent observations of output, we used a fourth 'Application' sub-network. Applications were used to measure both the relative nature of value creation (conventional v. novel) and risk (low, medium and high) associated with the cases, by comparing them with the relevant base cases. We compared two base cases and any follow-on steps by revisiting a series of interconnected consortia funding proposals. We also reviewed relevant scientific articles linked to specific digital innovation outputs (e.g., Daly et al., 2015; Brown et al., 2018). To dedude if the nature of value creation for specific applications continued to be conventional or were indeed novel, we explored how familiar respondents were with any novel performance parameters (i.e., had they observed and analysed a certain parameter at least once in a prior project). In terms of risk, we compared the same base cases with the sample and again deduced whether follow-on steps were deemed higher or lower risk, based on levels of variation observed for conventional and novel performance parameters.

\section{Empirical Observations}

In this section, we summarize our key empirical findings. First, we briefly outline our case sample and provide a summary of construct operationalization in terms of observed inputs in sections 4.1 and 4.2. We then summarize observed outputs and outcomes in section 4.3. and outline three empirical observations.

\subsection{Sequential Pathways}

\subsubsection{Pathway 1 (dPPI First)}

This pathway was representative of coordinated academic-industry initiatives aimed at transforming $3-4 \sigma$ sector performance to $5 \sigma$ levels. In other words, reducing large batch-tobatch variations (yields ranging anywhere from 93.3\% - 99.4\%) to achieve yields consistently $>99.9 \%$. Appendix 2 outlines a basic construct analysis for sequential dPPI cases 2-4. With molecule synthesis and crystallization unit operations considered as a dPPI 
case pair, Batch development (case 1) served as our base case in terms of traditional laboratory bench-scale 'make and test' approaches. As basic trade-offs and single-firm optimizations around procedural norms and policies were common-place here, we categorized our constructs (leverage, appropriability, and coherence) as low.

From this point of departure, we charted three dPPIs involving follow-on batch-dominant developments and their scale-up to material intensive commercial settings. Case 2 involved basic network initiatives that focused on reducing batch-to-batch variation in pilot and production settings. Here quality tended to be controlled through fixed process parameters and 'off-line' (destructive) end-product testing, with well-established rules on compliance to industry standards. So while there was some evidence of increasing leverage and engagement with selected partners, appropriability and coherence were categorized as low. Cases 3 and 4 involved dPPIs around batch processing that incorporated 'on-line' process analytics, and then a further transition from batch processing to hybrid (batch and continuous) modular systems, respectively. Here, more interdisciplinary networks were forming to collaborate on proof-of-concepts and research proposal development that focused on reducing batch-to-batch variation. With access to capabilities through increasing consortia engagements, leverage was categorized as high for the two cases. Case 3 centered on consortia-driven development of Quality-by-Design (QbD) principles. Here, digitalization efforts (in the form of advanced control and monitoring strategies) had the potential to eliminate or reduce the need for physical testing. With QbD implementation by individual firms evidenced, informing more targeted designs for their specific batch operations, the uptake of more 'on-line' analytics also introduced different types of trade-offs. Consortia members described these as being of a cross-sectional nature (i.e., risk versus return) and appropriability and coherence were categorized as medium in this case. Finally, case 4 involved dPPIs around combined batch and continuous processing schemes, supported by model-based predictive control involving 'real-time' process measurement. With emerging quality assurance standards being evaluated by consortia members for their 
own processes (appropriability seen as medium), leverage and coherence were categorized as high.

\subsubsection{Pathway 2 (dSCI First)}

This pathway was representative of specialized networks collaborating to improve noncompetitive aspects of demand and supply chains. Appendix 2 also outlines a basic construct analysis for sequential dSCl cases 6-8. With a focus on mandated serialization and clinical supply wastage, Make-to-stock supply (case 5) served as a base case in this pathway. Traditional initiatives here have typically involved optimizing (reducing) the high levels of inventory associated with traditional batch processing models. We again categorized our constructs (leverage, appropriability, and coherence) as low for this base case.

From this point of departure, we charted three dSCls involving follow-on developments in material intensive clinical and commercial supply settings. Case 6 involved experiments in commercial supply using printed electronics for basic compliance and quality monitoring which shipments are generally subjected to, e.g., environmental parameters (temperature and humidity). While coherence was regarded as low, mandated serialization for effective 'track and trace' of existing drug products has necessitated network formation around process and packaging redesign, and we categorized leverage and appropriability here as high. Case 7 was, in many ways, the polar opposite of our base case, with the development of lower volume dispersed 'factory' models. With consortia members looking to re-design supply chains for flexibility and agility in line with the development of more niche product variants targeted for sub-populations, we categorized leverage as high, and appropriability and coherence as medium. Finally, in this pathway, case 8 involved new trial designs to accelerate clinical and launch phases through 'just-in-time' operations. Regarding the current system, consortium members reported that it generally costs about $\$ 100$ million to run a clinical trial for a new drug. This is a significant burden given that $\sim 10 \%$ of drugs trialled only make it successfully through the development pipeline (DiMasi et al., 2016). As outlined in Appendix 2, we categorized leverage and coherence as high, and appropriability as medium. 


\subsection{Pathway 3 (Simultaneous)}

This pathway was representative of initiatives involving the development of dPPIs and dSCls in parallel. Appendix 3 outlines a basic construct analysis for eight simultaneous cases, where base cases 1 and 5 both served as our starting point in this pathway.

Case 9 was the first of two cases involving 'end-to-end' (E2E) proof-of-concept demonstrators (the other being case 16). Here, consortia were collaborating on concurrent workflow designs that integrated a series of dPPIs and dSCls. We categorized all of our three constructs as medium in this case. Case 10 involved mobile technology platforms and the development of continuous process equipment that could handle a range of chemistries for selected processes. Here, we observed consortia bringing together technology companies and their end users to deliver solutions based around a scale-up platform for synthesis and separation unit operations. Here, we categorized leverage and coherence as high, and appropriability as low. As outlined in Appendix 3, this categorization also applied to cases 12 (cloud-based software systems and 'smart label' interventions for effective trackand-trace) and 14 (new continuous processing technologies, including 3D-Printing, to increase the bioavailability of poorly soluble drugs).

In terms of agile processes, case 11 involved the design of equipment and emerging predictive models for the continuous filtration of molecules (product). Continuous filtration, although well established in other process industries at large scale, has received less attention on smaller scales suitable for medicines manufacture. Here, we categorized leverage and coherence as high, and appropriability as medium in this case. This categorization also applied to cases 13 (miniaturized manufacturing platforms, with 'realtime' process measurement and control for low volume and high variety contexts) and 15 (next-gen materials for robust packaging to keep medicines dry, secure and free from contaminants). Finally, the Digitalization Lab (case 16) was a proof-of-concept reference facility set up to facilitate the integration of continuous manufacturing equipment streams and dSCls (cases 9-15). Consortia members leveraged an open-access network of assets to quickly assess both firm-specific and consortia-specific projects (involving information 
exchanges between upstream with downstream processes) and evaluate alternative business models. We categorized leverage and coherence as high, and appropriability as medium in this case. In terms of consortia inputs, one respondent commented on simultaneous pathway projects involving the ReMediES programme:

"it is] one of the early examples of the pharmaceutical industry working together in collaborative $R \& D$, something it didn't have a record of doing before...you don't have to share your crown jewels ... there are a lot of areas where companies can work together... which can benefit the whole industry....[it] will deliver some early wins... and longer-term impacts...Things happened that nobody would have believed possible at the start". $\quad$ (CMAC Advisory Board Chair)

\subsection{Observed outputs and outcomes}

In line with our abductive approach, we used our data to describe phenomena we observed, in order to tease out tentative claims and narrow a range of possible explanations (Folger and Stein, 2017). Figures 3-6 summarizes observed outputs in terms of value creation and risk. A summary of outcomes (network performance) are outlined in Appendix 4. Further details are provided in on-line appendix A4. Patterns indicative of alternative dynamics, processes, and/or mechanisms were identified by filtering the dataset and we used contrastive reasoning to provide three empirical observations.

\subsubsection{Increasing Leverage}

We explored leverage effects by observing how integration sub-networks were coordinating projects in sequential and simultaneous pathways. We examined how unique memberspecific knowledge emerged and the nature of its uptake by other consortia members. We summarize outputs (through filtering) in Figure 3 and provide brief examples to support observed patterns using selected cases. In summary, increasing leverage (over the base cases) was associated with five cases where outputs were classified as conventional and of low risk. Four such cases were sequential, and one case was simultaneous. In contrast, increasing leverage was associated with nine cases where outputs were seen as novel and of higher risk. Here, two cases were sequential, while seven cases were simultaneous. 
For sequential case 2 (conventional value and low risk), outputs have continued to result in multiple - albeit small — changes to existing batch-based processing routines at molecule synthesis and crystallization.

CONVENTIONAL

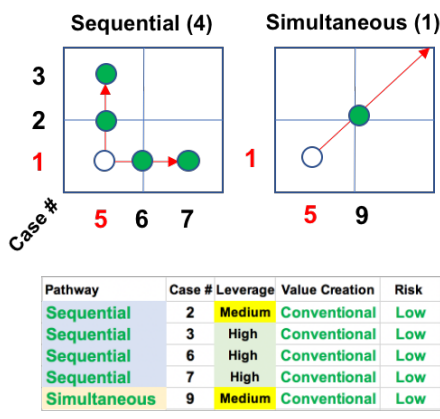

NOVEL

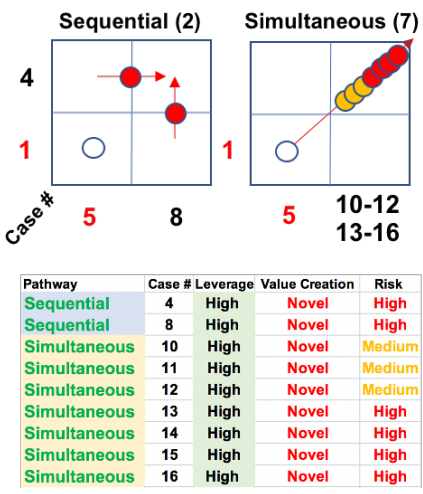

Figure 3. Increasing leverage cases - where outputs were deemed conventional or novel

A key barrier to transformational change is that many batch-mode routines continue to require multiple time-consuming 'work-up' operations post each step. Historically considered in isolation, these have served to negate some of the benefits of multiple incremental improvements made at molecule synthesis and crystallization in terms of quality (reducing variability in particle size). Unintended consequences are common-place for downstream unit operations from synthesis and crystallization in 'batch' manufacturing (i.e., variability in particle size being especially problematic at filtration steps, with serious implications for yield).

In contrast, for sequential case 4, 'data-driven' network-centric innovations have opened up the potential to transform the very nature of chemistry through better prediction of molecule-critical attributes and characteristics for rapid 'scale-up'. Industry-first breakthroughs involving elusive forms of target molecules have been achieved.

"...this has...rarely been achieved by design ...in this work such a designed process is not only achieved, but scaled to produce large quantities of an elusive [molecule] with enhanced physical properties" (Agnew et al., 2016, p. 7368)

Increased visibility on unique knowledge sources, involving such continuous-mode breakthroughs, has enabled consortia members to link outputs to promising network-centric innovations at other difficult downstream processes. For example, there were higher levels 
of confidence evidenced in integrating sequential case 4 outputs and simultaneous case 11 outputs. Here, consortia-led developments recently showcased the rapid determination of filtration parameters for new (and more challenging) molecules:

[With an industry-first predictive model for filterability]..."scale-up to continuous operation then represents lower risk... which has very different characteristics to manual laboratory process development or to current batch operations" (Ottoboni et al., 2019, p.381).

In conclusion, based on the patterns we observed, we can deduce the following: Empirical Observation 1: Increasing leverage (over the base cases) was associated with $67 \%$ of sequential cases and $12 \%$ of simultaneous cases where outputs were deemed conventional and of lower risk; conversely, increasing leverage was associated with $33 \%$ of sequential cases and $\mathbf{8 8 \%}$ of simultaneous cases where outputs were deemed novel and of higher risk.

\subsubsection{Increasing Appropriability}

We explored appropriability effects by observing 'knowledge mixes' in sequential and simultaneous pathways. That is, whether design rules sub-networks were reusing existing knowledge, or utilizing new consortia-derived knowledge, or a mix of both. We examined new routines and standards emerging from our cases and how consortia members also benefited from their contributions. We summarize outputs (through filtering) in Figure 4 and provide brief examples to support observed patterns using selected cases. In summary, increasing appropriability (over the base cases) was associated with four cases where outputs were seen as conventional and of low risk. Three cases here were sequential and one case was simultaneous. In contrast, increasing appropriability was associated with six cases where outputs were seen as novel and of higher risk. Here, two cases were sequential and four cases were simultaneous.

In assessing emerging regulations around serialization and digital standards, the easier (lower risk) option has seen single-firm optimizations that just follow the same rules (e.g., for compliance). Such internal initiatives tend to self-serve and were often characterised by transitory exchanges with the regulator in seeking approval for incremental modifications to existing routines. 
One area of focus has been the development of Quality-by-Design (QbD) principles by consortia where outcomes might result in less physical testing. While sequential dPPI progressions in digital production contexts have delivered some conventional value in terms of enabling data and information exchanges (case 3), implementing analytics linked to QbD principles for the 'real-time' release of products has long been an ambition (Yu et al., 2004) but not yet realized.

CONVENTIONAL
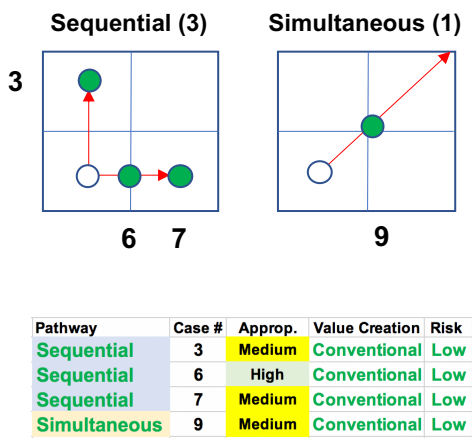

NOVEL

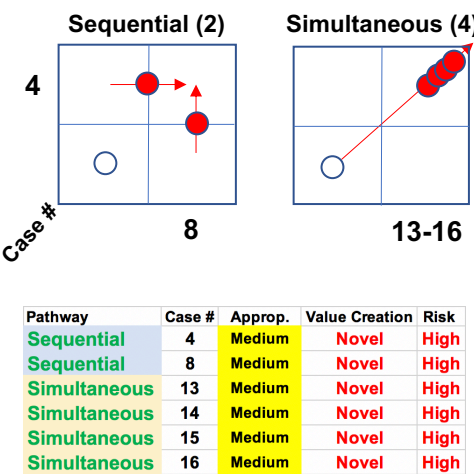

Figure 4. Increasing appropriability cases - where outputs were deemed conventional or novel

This is also being tackling in simultaneous pathways with case 9. Low risk feasibility demos - evaluating against existing standards - were commonplace in providing 'first steps' towards $\mathrm{QbD}$ through the predictive design of critical quality attributes (CQAs). An overarching workflow for the selection of overall process architecture, and setting of new standards, is a desired outcome here longer-term. However, follow-on experiments are required to address several limitations (e.g., the development of several workflows for unit operations downstream from molecule synthesis and crystallization and linking to E2E supply chain designs).

In contrast, novel dPPI outputs in both sequential and simultaneous pathways were being actively accessed with a view to utilizing new consortia-derived knowledge. In commenting on applying consortia feasibility learnings to problematic firm-specific batch processes, one partner saw the definition and prediction of 'ideal' process states, linked to 
cases 4 and 13, as something they could readily exploit in their firm; the other partner saw the same output as being more explorative in nature:

"the introduction of [novel] continuous seeding approaches developed with CMAC...into our scale-up operations allows for more consistent operation and predictable product properties"

(Development Fellow, Novartis)

"Overall it was a very valuable collaborative experience. I was really impressed that all the experiments in all equipment scenarios gave meaningful results...insights which have changed the way we think about our crystallization [unit operation].

(Technology Manager, Syngenta)

Similarly, for dSCls, sequential case 8 outputs have now made it possible to segment by product type (e.g., small molecule, formulation type, chemistry, stability), study design (complexity, shelf-life, phase and speed), customer demand profiles, technologies and risk profiles, in some cases. Emerging supply chain design rules and a more systems approach provided have improved conceptualisation and a modelling-based evaluation of supply chain configuration options. For consortia membership, this has enabled more 'customized' analysis in practice, with individual firms identifying what may be most critical for them in maximizing impact. Commenting on the benefits for his firm and for other consortia members, the project lead stated:

'We prototyped a new 'just-in-time' clinical pharmacy that can...support complex drug trials, thereby reducing costs, increasing responsiveness and enabling a more flexible and exploratory approach to clinical research...modelling of stock implications [has demonstrated the] potential benefit of...savings of $£ 10$ s of millions per year per company." (GSK Project Lead, ReMediES Clinical Platform)

In conclusion, based on the patterns we observed, we can deduce the following: Empirical Observation 2: Increasing appropriability (over the base cases) was associated with $50 \%$ of sequential cases and $12 \%$ of simultaneous cases where outputs were deemed conventional and of lower risk; conversely, increasing appropriability was associated with $33 \%$ of sequential cases and $\mathbf{5 0 \%}$ of simultaneous cases where outputs were deemed novel and of higher risk.

\subsubsection{Relationship of Coherence to Leverage and Appropriability}

Using observed inputs from all cases (see again Appendices 2 and 3), we charted transitions to new digitalization arrangements in sequential and simultaneous pathways. We then examined the role of coherence and the nature of trade-offs using the transition sub- 
network as the basis of observation. This transition sub-network was seen as the the linking mechanism between the integration and design rules sub-networks so we looked for patterns in terms of the relationship of coherence to leverage and appropriability respectively. Limitations of space constrain a full presentation of our comparative analysis, so we provide a summary in Appendix 4 and represent leverage—coherenceappropriability patterns graphically in Figure 5.

We observed that the nature of coherence appears to be fundamentally different in sequential and simultaneous pathways. Recall from cases 1 and 2, that traditional control strategies have been based around fixed recipes and profiles. For the sequential dPPI pathway, subsequent outputs relating to the quality control of selected unit operations are now enabling 'near-continuous' monitoring in practice with the adoption of advanced control 'on-line' strategies for specific processes.

\section{SEQUENTIAL}
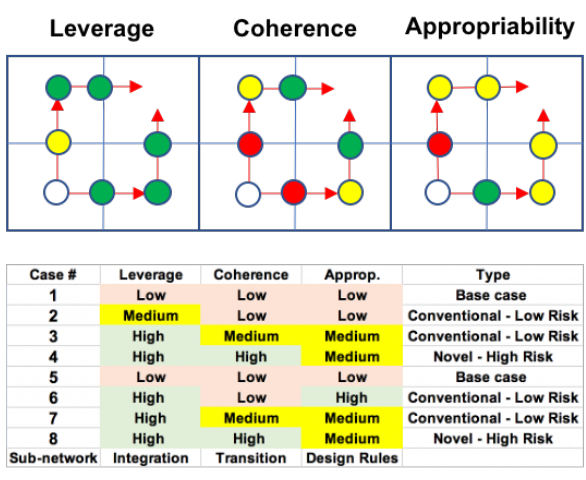

SIMULTANEOUS
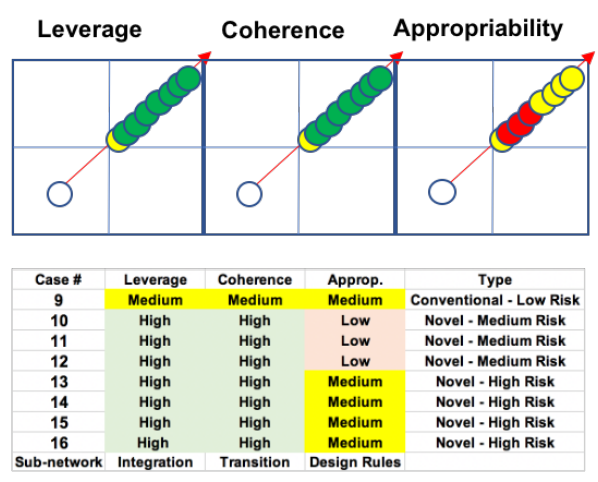

(Colours show level of ratio when compared to base cases 1 \& 5: red=low, yellow=medium, green=high)

Figure 5. Relationships between leverage, coherence, and appropriability for the case sample

Upstream and downstream operations (to molecule synthesis and crystallization) continue to be 'batch' or 'semi-continuous' and operated as decoupled operations often with independent coordination and governance mechanisms. In the other direction, sequential dSCI pathway outputs could be described as 'edge-to-edge' (they are doing this either 'at the edge', isolated from the main business or in ways which provide incremental improvements to their current activities) as opposed to 'end-to-end' in disrupting industry practices built around traditional 'make-to-stock' models. Here, for the most part, outputs 
have enabled a reduction in investments at risk via delayed decision requirements (spanning both development-clinical and production-commercial contexts).

In contrast, we observed the nature of know-how exchange to be fundamentally different in the simultaneous pathway. We observed a distinct shift from 'standard' dialogues and 'passive' interactions towards 'unique' conversations beyond the 'norm'. Predictive models were seen as essential building blocks for design rules that enabled transitions in simultaneous pathways. In meeting future market requirements to support lower-volume, high-variety niche products, simultaneous pathways also highlighted the need for new design rules around 'scale'. For example, for integrating smaller footprints — in setting up 'end-to-end' configurations that offered both flexibility in terms of production capacity, and speed in terms of 'scale-up' and 'scale-out'. In translating novel consortia know-how to their technology platform, one consortium member commented:

"This work... will be the basis for a core particle size control strategy in our continuous manufacturing platform...[it] offers superior particle size control in a shorter timeframe than traditional approaches. It is expected to save significantly on operator time and equipment use....The achievements were beyond expectations." (CMAC Industrial sponsor, Firm X)

Desired outcomes appeared as polar opposites to those of traditional high volume-low variety blockbuster business models and challenged the traditional location-decision logic (and that of the 'large batch' pharmaceutical plant). With new systems comes the need for new regulations, where regulatory confidence and internal buy-in was of paramount importance to consortia members. This was the goal in developing the reference facility (case 16) with an entirely digitalized and virtual approach to the design and launch of new products. Here, the traditional practice where products are taken through design, manufacturing and supply stages sequentially and separately, was replaced by a digital approach that enabled dPPIs and dSCls to be connected in a continuous E2E manner (cases 13-15). Rather than identifying and solving problems in isolation, manufacturing challenges were viewed holistically and managed as a consortium effort. According to one consortium project lead: 
"we needed to make the required transition real to people - including having a working production unit - so they could experience physically what can be achieved, and also embrace the changes and challenges associated with it' (Digitization lab lead, GSK)

Finally here, we observed how multiple partners are now required to deliver a wideranging set of applications. For example, future large-scale integration of 'point-of-care' solutions that might well require risk mitigations beyond some of the more traditional risk approaches identified for disruptive innovation. Reflecting on dPPIs and dSCls, designed around what he described as two overriding workstreams or 'platforms', one stakeholder commented on the consortium infrastructure:

'Before... [the consortium launched] collaborations were on [a] scale of each of our individual workstreams. We were advised that a programme with 24 partners, and the breadth of work...would be unmanageable. We managed to achieve this super-sizing by finding coherent themes, creating technical workpackages or Apps [applications], that sat within our two overriding workstreams for the clinical and commercial supply chains.'

(ReMediES programme co-designer and steering group member)

In conclusion, in examining the patterns between leverage, coherence, and appropriability for the case sample, we can deduce the following: Empirical Observation 3. Coherence and Leverage were aligned in $100 \%$ of the simultaneous cases and $33 \%$ of the sequential cases; Coherence and Appropriability work in the opposite direction, thus Coherence is associated with a variation in the moderation and mediation effect.

\section{Empirical Generalizations and Theoretical Interpretation}

Based on our summary of key empirical observations in section 4, we now look to give them plausible theoretical interpretations. This is common-place in theory-elaborating cases that rely on abductive reasoning, and in the post-factum interpretation of empirical data (Dubois and Gadde, 2002). First, we revisit our two research questions from section 2. What we can say with some confidence is: digitalization is forcing organizations to radically reconfigure, to decouple functional silo or single-firm optimizations, and adopt more 'platform-based' strategies involving both sequential and simultaneous pathways. The overarching interpretation of our observations is: digitalization is forcing firm sponsors and academic investigators who are structuring and managing consortia, and innovators operating within consortia-led projects, to engage in sensemaking and realignment. This is in line with 
Holmström et al (2020, p.731) who report that 'digitalization ...[is placing] conventional OSCM systems under stress'. Specifically, digital innovators concern themselves with how and where return on investments (ROls) and risk might drive pathway choices.

Hence, we give first insights on how consortia and their members organize and behave (often very differently) in transitioning, and offer a plausible mechanism that might inform firms when thinking about and managing certain environmental conditions. Recall RQ1 from section 2 (i.e., what are the risk implications of alternative pathways for conventional and novel outcomes?). Here, we offer two empirical generalizations:

Empirical Generalization 1: When value creation is conventional, sequential networkcentric digital innovations reduce risk when consortia members are able to highly leverage and integrate member-specific knowledge through appropriability; conversely, simultaneous network-centric digital innovations increase risk

Empirical Generalization 2: When value creation is novel, simultaneous network-centric digital innovations reduce risk when consortia members are able to highly leverage and integrate member-specific knowledge through appropriability; conversely, sequential network-centric digital innovations increase risk

In terms of RQ2 (i.e., what is the role of coherence in moderating and mediating the risk in these pathways?), we summarize our theoretical elaboration in variance theory form: Theoretical Elaboration 1: With sequential network-centric digital innovations, coherence both mediates and moderates the relationship between leverage and appropriability; with simultaneous network-centric digital innovations, coherence moderates the positive relationship between leverage and appropriability.

Figure 6 elucidates the dynamics by which 'knowledge mixes' may be adjusted over time in sequential and simultaneous pathways. This integrated view of our two empirical generalisations and one theoretical elaboration involving digital innovation pathways is consistent with Martin and Eisenhardt's stipulation on synthesis (2010) with its clear illustration of the underlying casual relations observed. In summary, sequential pathway mechanisms are both moderated and mediated by modular moves in transitioning (coherence). This is characterized by firms largely leveraging legacy systems with 'one side' 
digitalized first to ensure a 'rapid' test to determine success or failure. Firm- and consortialevel digital innovations can take many forms yet are based on conventional performance measures such as cost, quality, service, and dependability (and an ability to offer consistent or better improvements). With simultaneous pathways, network-centric digital innovations imply new technologies, standards, and radically different interpretations of performance measures. The risks in transition are being moderated by the presence of new networkcentric constructs (coherence). This pathway forces organizations to radically reconfigure and to rethink production/supply/regulatory networks and business models in tandem. For example, pre-competitive consortia have been 'proactive' in efforts to deliver novel systems and new regulations in a 'non-conventional' manner, as evidenced by 'end-to-end' digital demonstrators (linked to our cases) that do not always operate under current regulations. [This is also the case with emerging consortia working on Covid-19 vaccines. Here steps that are normally taken sequentially (and involve years) are being carried out simultaneously (in less than a year), e.g., managing large-scale clinical trials across 150 sites globally to qualify novel vaccine technologies, and scaling up manufacturing capabilities to produce billions of doses, and setting up the ultra cold-chain infrastructure to distribute vaccines, stored between -4 to -94 degrees Fahrenheit, around the world - all in paralle/].
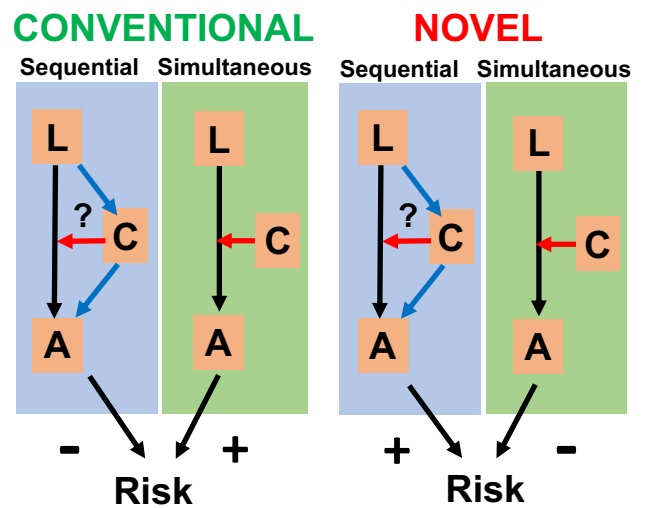

Figure 6. Alternative consortia-led digital innovation pathways: an integrated mechanism

As our research remit was about 'discovery' and conjecturable explanations, we recognize the difficulties in providing validated results or compelling evidence in places. This is because the strength of some of our claims are not 'certain or 'probable', as per deductive 
and inductive reasoning (Bamberger, 2018). So, in order to establish plausibility, we discuss and look to support the theoretical interpretations of our observations in sections 5.1-5.3.

\subsection{Leverage - making the most of 'consortia effects'}

Recalling our definition of leverage from section 2, what is common across all cases is that integration sub-network actors served as a 'backbone' in aligning network members and activities with the strategic goals of multiple consortia. In practical terms, individual firms were able to leverage resources, upwards of $50 \%$ in terms of direct activity, and by over $300 \%$ at a consortium level (Badman and Srai, 2018). One plausible explanation is that optimal consortia network structures were shaped based on the objectives of its members (Ahuja, 2009) and that 'network effects' were in play, evidenced by our increasing leverage cases (recall figure 3) and growth of consortia membership (lansiti and Levien, 2004). We also observed that the strength of these 'network effects' could vary dramatically in each pathway with consequences for value creation. This was consistent with Zhu and lansiti (2019) who outlined that the strength of these effects could vary but also change over time. Indeed, in this 8-year study, a form of 'relationship coordination' served to reduce the amount of load on academic-industry networks as a whole, which enabled consortia to shepherd choices over time and develop the necessary knowledge-integration capabilities in each pathway. This is consistent with the findings of Gardner et al. (2012) on teams who were successful in dynamically integrating members' resources into higher performance. This potential for variation only underscores the need to develop theory around the nature of value creation and risk in alternative pathways.

We also observed how increasing leverage cases were characterized by evidence of novel interactions and partnering models in fundamental and applied research. One plausible explanation here is that digitalization is now forcing firms to reconsider the overall business ecosystem. This is consistent with new modes of strategizing within and across firm boundaries (Holmström et al., 2020) where digital platforms and capabilities together are giving rise to business value created at the level of the ecosystem and shared by various 
co-contributors (Venkatraman et al., 2014). With familiarity and prior shared experiences positively impacting efficiency and quality of outputs (Staats, 2012), many firms have tended to collaborate with tried and trusted partners who may have delivered in the past (better the devil you know?). In consortia-led digitalization initiatives, firms have been exposed to new and previously untested partners but whose contributions may be more novel and potentially ground-breaking. This was particularly evident in simultaneous pathways, where 'unique SME-MNC pairings' aimed to deliver an increasingly diverse scope of applications at 'nonstandard' scales. As per appendix 4, our cases also highlighted the interchangeable roles of key actors with multiple combination options (e.g., 'integrator-allocator', 'pivot-coordinator', 'navigator-broker') based around emerging modes of innovation. This is consistent with the idea of 'distributed' innovation agency involving the dynamic and sometimes unexpected collection of actors engaging in the innovation process (Nambisan et al., 2017).

\subsection{Appropriability - out with the 'old' and in with the 'new'}

Recalling our definition of appropriability from section 2, our cases provide evidence of new routines and standards arising from digital innovations and their subsequent uptake. Some of which were initially based on uncertain and very risky hypothesis-driven developments. In practical terms, consortia members benefited greatly from adopting emerging design rules in their respective firms, which we outlined in section 4.3.2. With increasing confidence and trust (uptake of industry-first breakthroughs were seen to greatly reduce risk - see cases 10-12) came an increased pool of resources. This enabled innovators to influence further outcomes and turn follow-on or complementary projects (often chaotic at the outset) into "wins". One plausible explanation here is that with consortia-led projects, there are new, specific mitigation processes in play which helped to characterize and manage innovation risk profiles in each pathway. This is consistent with the idea of consortia as complex adaptive systems (Papadaki and Hirsch, 2013) 'facing endogenous risks as well as exogenous surprises that cannot always be anticipated in advance' (Lessard and Miller, 2001, p.9). We observed how consortia tackled decisions in the face of changing 
and uncertain environmental conditions and were open to rapid adjustments within sequential and simultaneous pathways. This invokes the idea of pathways as 'microenvironments' designed to drive stakeholder alignment and efficient adaptation strategies (Papadaki and Hirsch, 2013).

Traditional engagements involving conventional rules (for compliance) have typically resulted in transitory exchanges and once-off interactions between single firms and the UK regulator. Indeed, in section 4, we observed how dPPIs have led to multiple incremental changes to batch processing routines which have resulted in a myriad of 'exceptions' to existing rules. One plausible explanation here is moderating firm practices have tended to be easy to define and are reactive. This practice promotes numerous, yet minor changes, to routines to avoid resistance and mitigate organizational risk. As a result fleeting early successes (one-off case studies) involved more bounded innovation outcomes and processes, that were observed in traditional firm-firm activities observed pre-2011. This is consistent with Quinn (1978) who proposed that effective strategies tend to emerge iteratively. Firms probe the future, experiment, and learn from a series of incremental commitments.

Interestingly, the 'consortia effect' enabled active engagement and two-way information exchanges with the UK regulator in both pathways. In moving away from a traditional compliance discussion, collective conversations centered on up-front agreement and followon validation of novel consortium-led digital initiatives e.g., adaptive assembly-to-order solutions (case 8 ) in the sequential pathway. Our simultaneous pathway cases suggest less bounded innovation outcomes and processes, often reflected in new success criteria, as summarized in Appendix 4. The dynamic here, which might have been expected to be particularly challenging, was often focused on leveraging these industry-first breakthroughs and in promoting follow-on 'regulatory innovation' activities. This is consistent with precompetitive consortia enabling a 'whole-chain' view, in that they are 'fact-based rather than opinion-based' (Dooley, 2014, p.1108). As a result of those early successes, consortium 
members and regulators could choose to go 'all in' at times (increasingly more often than not) so that novel outcomes are maximized.

\subsection{Coherence - transitioning to new modes of operation}

Recalling our definition of coherence from section 2, our cases provide evidence of critical communications and cross-sectional activities at higher levels of abstraction being managed in both sequential and simultaneous pathways. In practical terms, the 'Transition' subnetwork looked to revise traditional practices and 'transition' using digitalization capabilities developed through consortia engagements. Here, new rules derived by multiple engagements involving multiple partners and based around platform-based thinking, and other 'unconventional' practicalities, were in evidence. This invoked the idea of 'boundary objects', shared and shareable across different problem solving contexts (Star, 1989 in Carlile, 2002). Here, despite substantial differences between our consortia members across various disciplines, they nevertheless were very successful in cooperating and transitioning to create value and mitigate risks. The mode of innovation was also changing here, evidenced by firms abandoning 'batch' development in favour of 'continuous' approaches. One plausible explanation here is that firms have traditionally focused either on the process (with limited attention to the innovation outcome) or the innovation outcome (with limited attention to the innovation process) (Nambisan et al., 2017). As a result, moderating firm practices were often sequential, avoiding those actions that are loaded with risk in transitioning to a future (desired) state. However, modifications can be immediate in instances where no regulatory changes are needed. With digitalization, dependencies between processes and outcomes appear more complex and dynamic, so may constrain or shape action, value, and choices along different pathways (Carlile, 2003). Applying this to sequential and simultaneous pathways, these boundary objects - objects that work to establish a shared context that "sits in the middle" - are somewhat different. They are better defined in sequential pathways given its temporal nature, but more blurred in simultaneous pathways as interfaces are novel and difficult to scale. This is consistent with Dougherty and 
Dunne (2012) who proposed that digitalization, in drug discovery contexts, led to a reorganization of the innovation focus. The creation of a new and necessary set of activities here had implications for innovation outcomes - the effects of which were all unintended.

\section{Managerial Implications}

The theoretical propositions described in section 5 have significant managerial implications. From a strategic operations management perspective, this research provides insights on consortia-based pre-competitive collaborations in the context of innovations involving the application of multiple technologies. These technologies traverse product-process development and supply chain innovation. We expand on these insights below in terms of project portfolio and subsequent consortia development, value appropriation and risk mitigation. At the operational level, the central managerial insight that we identify is when to adopt the sequential pathway and when the simultaneous pathway is more appropriate, primarily in the context of risk mitigation. This presents a major dilemma for innovators, in terms of how firms handle this technology configuration challenge i.e., sequential (on the surface a more steady approach but loaded with the risk of never arriving at the end-goal within a reasonable timeframe) versus 'all-in' simultaneous transitions (often seen as loaded with risk but with the promise of breakthrough outcomes). We can draw the following insights and recommendations from our observations.

As per Chandrasekaran et al., (2015), project and organizational contexts affect performance and are dependent on the type of project. First, at a strategic management level, we observe the 'flywheel effect' of multiple pre-competitive collaborations that followon from each other as capabilities and trust develops across partners, underpinned by early successes and results that then shape longer-term ambitions. The evidence suggests that both radical and incremental projects are required to sustain consortia, and this mixed portfolio approach is necessary to maintain 'momentum'. However, firms need to position themselves to be able to switch from exploiting existing knowledge to exploring new knowledge (March, 1991) and vice-versa, based on business model needs. This finding 
suggests that both sequential and simultaneous pathways play a critical role in sustaining consortia, despite their very different risk profiles. Further, firms and their networks need to collectively assess strategies and set up a supporting consortium infrastructure (or a set of platforms) to deliver network performance —-that is, assessing different pathway choices for value creation and risk at a project level, involving both shorter term ROI projects as well as platform-level projects that will support the 'next round' of pre-competitive collaborations. In sequential pathways, 'use cases' have typically been the modus operandi but are often characterised by 'tactical gains' (e.g., new technology adoptions at one process step that is implemented for just one product type) as a means of getting internal buy-in within an organization, and based on a near term ROI justification. Hence, 'attractive' cases often determine the pathway choice which can result in short-term incremental activity (but possibly never the long term 'strategic intent' end-point). In contrast, simultaneous pathways require the development of hypotheses and a future vision at a strategic level. Immediate $\mathrm{ROI}$ is not always apparent as investing in e.g., a platform requires many players and pooled resources (intellectual and financial). The consortium effect serves to 'de-risk the risk conversation', which helps consortium partners sell the hypotheses to internal stakeholders in their respective organizations. The consortia model thus leverages individual investments with a multiplier effect through the pooling of resources. Further, for novel innovations, the regulatory innovation dynamic which might be expected as particularly challenging (and prohibitive for single firms), is transformed; the regulator role moves from a compliancecentric single company discussion, to a 'co-creation' platform-building activity.

The adoption of multiple pathways within consortium models also has significant managerial implications. In our study the multiple projects 'portfolio mix' provides flexibility, each pathway and associated project type with their distinct management approach, itself providing a risk-hedging form of mitigation. As projects progress, resources can be redirected based on results and re-evaluation of project potential, with project learnings informing on-going workstream or platform design. This classification of conventional and novel projects is in itself a significant management takeaway; suggesting that perhaps 
consortia models are essential mechanisms for novel-simultaneous execution projects, but perhaps counter intuitively, also for conventional-sequential execution projects to leverage knowledge and appropriability for this particular innovation-intensive context. Perhaps also a surprising finding is the 'all-in' approach is most appropriate from a risk mitigation perspective for novel 'moon-shot' projects, avoiding the bureaucracy of multiple stage-gate ROI centric reviews, a restrictive regulatory regimen and consequent extended timelines. The role of institutional players becomes significant for the success of these more radical projects, be they the technology providers (universities and SMEs), funding bodies (government), first adopters (MNCs), and regulatory bodies, making their participation in such consortia vital.

\section{Conclusion}

Consortia members often have very different ideas when it comes to outcomes and risk. Some are ambitious with a long-term vision, while others focus on short-term incremental gains. This paper outlines three alternative digital innovation pathways that firms navigated in the context of pre-competitive consortia. Over eight years, we were privy to the unique issues and difficult choices they had to make. For example, what pathway do you take if your ultimate goal is a conventional or novel outcome? In practice, this is a major dilemma for those structuring and operating within consortia-led innovation projects. Hence, we offer an integrated mechanism that might inform when to adopt a sequential pathway, and when the simultaneous pathway is more appropriate.

This paper has elaborated, through empirical case studies, on the orchestration processes of network-centric innovations (Nambisan and Sawhney, 2011). We offer midrange theoretical insight and propositions for further empirical research, and recognize that such studies are required to explore some of the elicit tentative claims we make. However, these have opened up promising research opportunities that we are pursuing. First, replication and extension in other ecosystems will help to extend our collective understanding of sequential and simultaneous digital innovation pathways. If future 
research, via case-based and survey-based research designs, in other contexts support similar arguments we can conclude our results - obtained through abductive reasoning could support inductive studies to confirm generalizable outcomes. Our observations on sequential and simultaneous pathways, and allied data-driven decision-making consistent with Guha and Kumar (2017), offer a second possibility for future research in terms of optimization research. Finally, it will be important to better understand knowledge transfer mechanisms, the role of boundary objects and allied operational routine options (Carlile and Rebentisch, 2003), i.e., are new types of boundary objects needed to facilitate the directional shifts required in alternative pathways?

Finally, molecules can be divided into two classes - small and large - which significantly impacts technology platform choices. Hence, one limitation was a focus on dPPIs and dSCls involving small molecules only. This is an opportunity as competition begins to shift based on new business models e.g., impending batch-to-continuous-conversion tipping point evidenced by our sample; strategic shifts towards large molecules (e.g., biologics and vaccines) and drug-device combinations (Waltz, 2014). Hence, we are considering the implications of our propositions as firms seek to make sense of future R\&D investments.

\section{References}

Agnew, L.R., Cruickshank, D.L., McGlone, T., Wilson, C.C., 2016. Controlled production of the elusive metastable form II of acetaminophen (paracetamol): a fully scalable templating approach in a cooling environment. Chemical Comms. 52, 7368-7371.

Ahuja, G., 2000. Collaboration networks, structural holes, and innovation: A longitudinal study. Administrative Science Quarterly. 45 (3), 425-455.

Badman, C., Trout, B.L., 2015. Achieving Continuous Manufacturing. May 20-21, 2014 Continuous Manufacturing Symposium. J. Pharm Sciences. 104 (3), 779-780.

Badman, C., Srai, J.S., 2018. ReMediES: collaborative research in action. Cambridge.

Bamberger, P.A. 2018. AMD - Clarifying What We're About and Where We're Going. Administrative Science Discoveries. 4 (1), 1-10.

Carlile, P.R., Rebentisch, E.S. 2003. Into the Black Box: The Knowledge Transformation Cycle. Management Science. 49 (9), 1180-1195.

Chandrasekaran, A., K. Linderman, R. Schroeder. 2015. The role of project and organizational context in managing high-tech R\&D projects. POM 24, 560-586.

Chandrasekaran, A., Linderman, K., Sting, F., and Benner, M. 2016. "Managing R\&D project shifts in high-tech organizations: Multi-method study." POM. 25(3), 390-416 
Corbin, J., Strauss, A. 1990. Grounded Theory Research: Procedures, Canons, and Evaluative Criteria. Qualitative Sociology, Vol. 13, No. 1, 3-21.

Dhanaraj, C., Parkhe, A. 2006. Orchestrating Innovation Networks. Administrative Science Review. 31 (3), https://doi.org/10.5465/amr.2006.21318923

Daly, R., Harrington, T.S., et al. 2015. Inkjet printing for pharmaceutics - A review of research and manufacturing. Int J Pharmaceutics, 494 (2), 554-567.

DiMasi, J.A., Grabowski, H.G., Hansen, R.W. 2016. Innovation in the Pharmaceutical Industry: New Estimates of R\&D Costs. Journal of Health Economics. 47, 20-33.

Dubois, A., Gadde, L.E. 2002. Systematic combining: an abductive approach to case research. Journal of Business Research, 55, 553-60.

Edmondson, A., McManus, S., 2007. Methodological Fit in Management Field Research. Academy of Management Review. 32, 1155-1179.

Eisenhardt, K.M., 1989. Building theories from case study research. Academy of Management Review. 14 (4), 532-550.

Eisenhardt, K.M., Graebner, M.E., 2007. Theory building from cases: opportunities and challenges. Academy of Management Journal. 50, 25-32.

FDA. 2019. https://www.fda.gov/drugs/drug-supply-chain-security-act-dscsa/are-you-ready-drug-supply-chain-security-act.

Folger, R., Stein, C. 2016. Abduction 101: Reasoning processes to aid discovery. Human Resource Management Review, 27(2), 306-315

Frølund, L., Riedel, M. 2018. Strategic Industry-University Partnerships. Academic Press.

Gardner, H., Gino, F., Staats, B.R. 2012. Dynamically Integrating Knowledge in Teams: Transforming Resources into Performance. Academy of Mgmt J, 55(4), 998-1022.

Gnyawali, D.R., Park, B-Y., 2011. Co-opetition between giants: Collaboration with competitors for technological innovation. Research Policy. 40 (5), 650-663.

lansiti, M., Levien, R., 2004. The keystone advantage: what the new dynamics of business ecosystems mean for strategy, innovation, and sustainability. Harvard Bus. Press.

IBM Corp, 2001. SPSS two-step cluster analysis. https://www.ibm.com/support/knowledgecenter/bs/SSLVMB 24.0.0/spss/base/idh twostep main.html.

Joglekar, N.R., Davies, J., Anderson, E.G., 2016. The role of industry studies and public policies in production and operations management. POM. 25 (12), 1977-2001.

Ketokivi, M. 2006. Elaborating the Contingency Theory of Organizations: The Case of Manufacturing Flexibility Strategies. POM. 15 (2), 215-228.

Ketokivi, M., Choi, T. 2014. Renaissance of case research as a scientific method. JOM. 32 (5), 232-240.

Leclerc, O., Smith, J., 2018. Will digital platforms transform pharmaceuticals? McKinsey Quarterly. August edition. 2-6.

March, J.G. 1991. Exploration and Exploitation in Organizational Learning. Organization Science. 2 (1), 71-87

Martin, J.A., Eisenhardt, K.M. 2010. Rewiring: Cross-business-unit collaborations in multibusiness organizations. Academy of Mgmt Journal. 53(2), 265-301.

Munos, B., 2009. Lessons from 60 years of pharmaceutical innovation. Nature Reviews Drug Discovery. 8 (12), 959-68.

Nambisan, S., Sawhney, M., 2011. Orchestration Processes in Network-Centric Innovation: Evidence From the Field. Academy of Mgmt Perspectives. 25 (3), 40-57. 
Nambisan, S., Lyytinen, K., Majchrzak, A., Song, M., 2017. Digital Innovation Management: Reinventing innovation management research in a digital world. MIS Quarterly. 41 (1), 223-238.

Ottoboni, S., et al., 2019. Development of a Novel Continuous Filtration Unit for Pharmaceutical Process Development and Manufacturing. J. Pharm Sc. 108 372-381.

Papadaki, M., G. Hirsch, G. 2013. Curing consortium fatigue. Sci. Transl. Med. 5 1-3.

Parraguez, P., Eppinger, S., Maier, A., 2016. Characterizing Design Process Interfaces as Organization Networks: Insights for Engineering Systems Management. Systems Eng. 19 (2), 158-173.

Pathak, S., Wu, Z., Johnston, D., 2014. Toward a structural view of co-opetition in supply networks. JOM. 32 (5), 254-267.

Patton, M.Q. 1990. Qualitative evaluation and research methods. 2nd ed., Newbury Park, CA. Sage

Pich, M.T., Loch, C.H., De Meyer, A. 2002. On Uncertainty, Ambiguity, and Complexity in Project Management. Management Science. 48 (8), 1008-1023.

Powell, W.W., Koput, K.W., Smith-Doerr, L., 1996. Interorganizational collaboration and the locus of innovation: Networks of learning in biotechnology. Admin Sci Q. 41 (1), 116-145.

Srai, J.S., Badman, C., Krumme, M., Futran, M., Johnston. C., 2015. Future Supply Chains Enabled by Continuous Processing-Opportunities and Challenges,. J Pharm Sci 104 (3), 840-849.

Srai, J.S., Harrington, T.S., Tiwari, M.K., 2016. Characteristics of redistributed manufacturing systems: a comparative study of emerging industry supply networks. Int J Prod Res. 54 (23), 6936-6955.

Staats, B.R. 2012. Unpacking Team Familiarity: The Effect of Geographic Location and Hierarchical Role. POM. 21(3), 619-635.

Thomke, S. 2003. Experimentation Matters: Unlocking the Potential of New Technologies for Innovation. Boston, MA: Harvard Business School Press.

Venkatraman, N., El Sawy, O., Pavlou, P., Bharadwaj, A., 2014. Theorizing digital business innovation: platforms and capabilities in ecosystems. SSRN. Accessed 18 July'18.

Waltz, E., 2014. It's official: biologics are pharma's darlings. Nature Biotechnology, 32, 117.

Yu, L.X., Lioberger, R.A., Raw, A.S., D'Costa, R., Wu, H., Hussain, A.J., 2004. Applications of process analytical technology to crystallization processes. Adv Drug Delivery Rev. 56(3), 349-369.

Yin, R.K. 2009. Case Study Research: Design and Methods. 4th ed. Thousand Oaks, CA: Sage.

Zhu, F., lansiti, M. 2019. Why Some Platforms Thrive and Others Don't. Harvard Business Review. https://hbr.org/2019/01/why-some-platforms-thrive-and-others-dont. 4 July, 2019.

Appendix 1. UK consortia in this study

\begin{tabular}{|l|l|}
\hline Consortium & Website \\
\hline Dial-a-Molecule Grand Challenge Network & http://generic.wordpress.soton.ac.uk/dial-a-molecule/ \\
\hline $\begin{array}{l}\text { CIM CMAC (Centre for Innovative Manufacturing in Continuous } \\
\text { Manufacturing and Crystallization) }\end{array}$ & https://www.cmac.ac.uk \\
\hline $\begin{array}{l}\text { ReMediES (Reconfiguring Medicines End-to-End Supply) } \\
\text { programme }\end{array}$ & $\underline{\text { https://remediesproject.com }}$ \\
\hline $\begin{array}{l}\text { ADDoPT (Advanced Digital Design Transforming Pharma } \\
\text { Development and Manufacture) programme }\end{array}$ & $\underline{\text { https://www.addopt.org }}$ \\
\hline $\begin{array}{l}\text { CMAC Hub (Future Continuous Manufacturing and Advanced } \\
\text { Crystallization Research Hub) }\end{array}$ & $\underline{\text { https://www.cmac.ac.uk; }}$ \\
\hline
\end{tabular}


Appendix 2. Sequential case profiles with summary of observed inputs (basic construct analysis)

\begin{tabular}{|c|c|c|c|c|}
\hline \multirow[t]{2}{*}{ \# } & \multirow{2}{*}{$\begin{array}{l}\text { Case overview } \\
\text { Focus }\end{array}$} & \multicolumn{3}{|c|}{ OBSERVED INPUTS (and basis of observation) } \\
\hline & & $\begin{array}{l}\text { Leverage Ratio } \\
\text { Integration sub-network }\end{array}$ & $\begin{array}{l}\text { Appropriability Ratio } \\
\text { Design rules sub-network }\end{array}$ & $\begin{array}{l}\text { Coherence Ratio } \\
\text { Transition sub-network }\end{array}$ \\
\hline 1 & $\begin{array}{l}\text { Batch development } \\
\text { Traditional lab-scale 'make and } \\
\text { test' approaches }\end{array}$ & $\begin{array}{l}\text { Low } \\
\text { Limited and embryonic network } \\
\text { structures; basic product definitions; } \\
\text { Focus on 'in-house validation' initiatives }\end{array}$ & $\begin{array}{l}\text { Low } \\
\text { Operating in line with existing routines and } \\
\text { standards; single-firm optimizations }\end{array}$ & $\begin{array}{l}\text { Low } \\
\text { Classic trade-offs of speed versus } \\
\text { accuracy (temporal) }\end{array}$ \\
\hline 2 & $\begin{array}{l}\text { Batch processing with off-line } \\
\text { analytics } \\
\text { Process control through fixed } \\
\text { parameters }\end{array}$ & $\begin{array}{l}\text { Medium } \\
\text { Fragmented single-firm networks; } \\
\text { commercial strategies in place }\end{array}$ & $\begin{array}{l}\text { Low } \\
\text { Compliance to well-established industry } \\
\text { standards; fixed parameters }\end{array}$ & $\begin{array}{l}\text { Low } \\
\text { Conventional end-of-line testing; } \\
\text { temporal trade-offs as default at scale }\end{array}$ \\
\hline 3 & $\begin{array}{l}\text { Batch processing with on-line } \\
\text { analytics } \\
\text { Quality-by-design }(\mathrm{QbD}) \text { principles } \\
\text { with novel analytics }\end{array}$ & $\begin{array}{l}\text { High } \\
\text { Networks formed around collaborative } \\
\text { research }\end{array}$ & $\begin{array}{l}\text { Medium } \\
\text { Emerging QbD principles for selected } \\
\text { batch operations }\end{array}$ & $\begin{array}{l}\text { Medium } \\
\text { Targeted experimental designs; Risk } \\
\text { v. return (cross-sectional) trade-offs }\end{array}$ \\
\hline 4 & $\begin{array}{l}\text { Hybrid processing systems with } \\
\text { on-line analytics } \\
\text { Model-based predictive control }\end{array}$ & $\begin{array}{l}\text { High } \\
\text { Virtual integrated networks; access to } \\
\text { consortia-wide capabilities for members }\end{array}$ & $\begin{array}{l}\text { Medium } \\
\text { Existing (quality control) and emerging } \\
\text { (quality assurance) standards possible }\end{array}$ & $\begin{array}{l}\text { High } \\
\text { 'Real-time' comparisons; 'ideal' } \\
\text { process states predicted and defined }\end{array}$ \\
\hline 5 & $\begin{array}{l}\text { Make-to-stock supply } \\
\text { High volume centralised models }\end{array}$ & $\begin{array}{l}\text { Low } \\
\text { Centralized single-firm arrangements }\end{array}$ & $\begin{array}{l}\text { Low } \\
\text { Compliance to well-established industry } \\
\text { standards; fixed parameters }\end{array}$ & $\begin{array}{l}\text { Low } \\
\text { Traditional cost versus quality } \\
\text { (temporal) trade-offs }\end{array}$ \\
\hline 6 & $\begin{array}{l}\text { Printed electronics } \\
\text { Prototypes for 'track-and-trace' }\end{array}$ & $\begin{array}{l}\text { High } \\
\text { Network formation around process and } \\
\text { packaging re-design }\end{array}$ & $\begin{array}{l}\text { High } \\
\text { Mandated serialization; high potential in } \\
\text { replacing industry standard }\end{array}$ & $\begin{array}{l}\text { Low } \\
\text { Basic quality monitoring for } \\
\text { compliance }\end{array}$ \\
\hline 7 & $\begin{array}{l}\text { Localised on-demand supply } \\
\text { Low volume dispersed models }\end{array}$ & $\begin{array}{l}\text { High } \\
\text { Specialists re-designing supply chains for } \\
\text { flexibility and agility and located closer to } \\
\text { the points of use }\end{array}$ & $\begin{array}{l}\text { Medium } \\
\text { Increasing convergence of multiple digital } \\
\text { technologies in clinical (Advanced } \\
\text { Manufacturing Technologies-Packaging) }\end{array}$ & $\begin{array}{l}\text { Medium } \\
\text { Targeted experimental designs; Risk } \\
\text { v. return (cross-sectional) trade-offs }\end{array}$ \\
\hline 8 & $\begin{array}{l}\text { Adaptive assembly-to-order } \\
\text { solutions } \\
\text { Trial designs to accelerate clinical } \\
\text { phases }\end{array}$ & $\begin{array}{l}\text { High } \\
\text { Consortium approach enabling novel } \\
\text { engagements with regulators }\end{array}$ & $\begin{array}{l}\text { Medium } \\
\text { Customised product design } \\
\text { ('personalisation' in terms of country- } \\
\text { clinic-individual) }\end{array}$ & $\begin{array}{l}\text { High } \\
\text { Specialist risk partner focus on risk } \\
\text { versus high return trade-offs }\end{array}$ \\
\hline
\end{tabular}


Appendix 3. Simultaneous case profiles with summary of observed inputs (basic construct analysis)

\begin{tabular}{|c|c|c|c|c|}
\hline \multirow[b]{2}{*}{ \# } & \multirow[b]{2}{*}{$\begin{array}{l}\text { Case overview } \\
\text { Focus }\end{array}$} & \multicolumn{3}{|c|}{ OBSERVED INPUTS } \\
\hline & & $\begin{array}{l}\text { Leverage Ratio } \\
\text { Integration sub-network }\end{array}$ & $\begin{array}{l}\text { Appropriability Ratio } \\
\text { Design rules sub-network }\end{array}$ & $\begin{array}{l}\text { Coherence Ratio } \\
\text { Transition sub-network }\end{array}$ \\
\hline 9 & $\begin{array}{l}\text { Digital workflow } \\
\text { E2E Proof-of-Concept demonstrator }\end{array}$ & $\begin{array}{l}\text { Medium } \\
\text { Select consortia partners } \\
\text { experimenting with concurrent designs }\end{array}$ & $\begin{array}{l}\text { Medium } \\
\text { Compliance to existing standards as a } \\
\text { benchmark for comparable performance }\end{array}$ & $\begin{array}{l}\text { Medium } \\
\text { Prototype framework developed } \\
\text { for E2E business case transitions }\end{array}$ \\
\hline 10 & $\begin{array}{l}\text { Plug-and-Play } \\
\text { Mobile technology platforms with open } \\
\text { access }\end{array}$ & $\begin{array}{l}\text { High } \\
\text { Collaborative models gaining traction } \\
\text { over differentiated approaches }\end{array}$ & $\begin{array}{l}\text { Low } \\
\text { Qualification using existing standards; } \\
\text { validation demos for select chemistries }\end{array}$ & $\begin{array}{l}\text { High } \\
\text { Full-scale transitions via modules } \\
\text { available at lab and pilot scale }\end{array}$ \\
\hline 11 & $\begin{array}{l}\text { Continuous Filtration } \\
\text { Agile processes for smaller scale } \\
\text { manufacture }\end{array}$ & $\begin{array}{l}\text { High } \\
\text { Network actors demonstrating benefits } \\
\text { via exemplar case studies }\end{array}$ & $\begin{array}{l}\text { Medium } \\
\text { Comparable performance (if not better) } \\
\text { versus manual best practice and } \\
\text { standards in process development }\end{array}$ & $\begin{array}{l}\text { High } \\
\text { Defining design space in terms of } \\
\text { critical material attributes and } \\
\text { process parameters }\end{array}$ \\
\hline 12 & $\begin{array}{l}\text { Next-Generation RFID } \\
\text { 'Smart label' interventions }\end{array}$ & $\begin{array}{l}\text { High } \\
\text { Partnerships teasing out explicit links } \\
\text { with non-consortium partners }\end{array}$ & $\begin{array}{l}\text { Low } \\
\text { Basic compliance and anti-counterfeiting } \\
\text { focus }\end{array}$ & $\begin{array}{l}\text { High } \\
\text { 'Real-time' data for enhanced } \\
\text { visibility }\end{array}$ \\
\hline 13 & $\begin{array}{l}\text { Micro-factories } \\
\text { Flexible mobile platforms for low } \\
\text { volumes }\end{array}$ & $\begin{array}{l}\text { High } \\
\text { Network formation around micro- } \\
\text { factory archetypes and concepts }\end{array}$ & $\begin{array}{l}\text { Medium } \\
\text { Precision medicine driving smaller } \\
\text { volume production and supply models }\end{array}$ & $\begin{array}{l}\text { High } \\
\text { Novel network designs; Digital } \\
\text { twins; elimination of multiple steps }\end{array}$ \\
\hline 14 & $\begin{array}{l}\text { Continuous Extrusion and 3D-Print } \\
\text { Agile processes for advanced delivery }\end{array}$ & $\begin{array}{l}\text { High } \\
\text { Consortia-led feasibility studies } \\
\text { involving some demonstration of } \\
\text { structured and controlled drug-release } \\
\text { systems }\end{array}$ & $\begin{array}{l}\text { Low } \\
\text { Looking to existing standards as } \\
\text { benchmarks; emerging predictive } \\
\text { models in secondary processing (drug } \\
\text { product) }\end{array}$ & $\begin{array}{l}\text { High } \\
\text { Defining design spaces in terms of } \\
\text { critical material attributes and } \\
\text { process parameters }\end{array}$ \\
\hline 15 & $\begin{array}{l}\text { Next-Generation product concepts } \\
\text { Developing new types of packaging }\end{array}$ & $\begin{array}{l}\text { High } \\
\text { Network actors demonstrating benefits } \\
\text { via exemplar case studies with new } \\
\text { materials and processing techniques } \\
\end{array}$ & $\begin{array}{l}\text { Medium } \\
\text { Potential to replace standard material for } \\
\text { a wide range of medicines }\end{array}$ & $\begin{array}{l}\text { High } \\
\text { Digital standard operating } \\
\text { procedures; Links to digital leaflets } \\
\text { and mobile phone apps } \\
\end{array}$ \\
\hline 16 & $\begin{array}{l}\text { Digitalization Lab } \\
\text { E2E Proof-of-Concept demonstrator }\end{array}$ & $\begin{array}{l}\text { High } \\
\text { Open access asset network for use by } \\
\text { consortia membership }\end{array}$ & $\begin{array}{l}\text { Medium } \\
\text { Data and information to evaluate } \\
\text { business model-changing shifts }\end{array}$ & $\begin{array}{l}\text { High } \\
\text { Rapid assessment of } \\
\text { experimentation }\end{array}$ \\
\hline
\end{tabular}


Appendix 4. Summary of outcomes and the relationship of coherence to leverage and appropriability

\begin{tabular}{|c|c|c|c|}
\hline Construct & Leverage & Coherence & Appropriability \\
\hline $\begin{array}{c}\text { Basis of } \\
\text { observation }\end{array}$ & Integration sub-network & Transition sub-network & Design Rules sub-network \\
\hline $\begin{array}{l}\text { Sequential } \\
\text { pathway } 1 \\
\text { (dPPI first) } \\
\text { Cases } \\
1-4\end{array}$ & $\begin{array}{l}{ }^{*} \text { Principle nodes co-developing 'data- } \\
\text { driven' experimentation and brokering } \\
\text { access to potential end-user groups, as } \\
\text { pairs of 'pivots' and 'navigators' related to } \\
\text { their specialisms; } \\
{ }^{*} \text { Virtual networks emerging around 'assets } \\
\text { and capabilities' made accessible by } \\
\text { consortia membership to wider } \\
\text { communities }\end{array}$ & $\begin{array}{l}\text { * Principle nodes facilitating moves towards modelling- } \\
\text { based design of drug products and manufacturing } \\
\text { processes; } \\
\text { * Experimentation goes beyond traditional DoE by } \\
\text { leveraging consortiua expertise to support delivery of } \\
\text { 'targeted' molecules and 'robust' processes; } \\
\text { * Sequential changes in predictive capabilities now } \\
\text { enabling 'real-time' comparison of batches against } \\
\text { 'ideal' process states for selected processes }\end{array}$ & $\begin{array}{l}\text { * Principle nodes supporting increased } \\
\text { information flows between digital design activities } \\
\text { and full-scale manufacturing processes; } \\
\text { * Experiments around high-volume low-variety } \\
\text { segments continue to dominate; } \\
\text { * 'Quality-by-Design' (QbD) principles have } \\
\text { emerged over time which are mitigating but linear }\end{array}$ \\
\hline $\begin{array}{l}\text { Sequential } \\
\text { pathway } 2 \\
\text { (dSCI first) } \\
\text { Cases } \\
5-8\end{array}$ & $\begin{array}{l}\text { * Principle nodes co-developing } \\
\text { 'segmentation-driven' experimentation } \\
{ }^{*} \text { New 'outcome-based' product delivery } \\
\text { models coordinated by both clinical and } \\
\text { commercial central nodes } \\
{ }^{*} \text { Specialised sub-networks commissioned } \\
\text { to design clinical trials and protocols with } \\
\text { built-in flexibility and agility }\end{array}$ & $\begin{array}{l}\text { * Principle nodes facilitating enhancement of traditional } \\
\text { 'make-to-stock' supply models using 'adaptive' } \\
\text { approaches enabled through digital information } \\
{ }^{*} \text { Sequential changes in process and packaging } \\
\text { redesign supporting potential for more Quality } \\
\text { Assurance (QA) dominant product releases in certain } \\
\text { cases } \\
{ }^{*} \text { Data systems reconfigured for improved traceability } \\
\text { and compliance monitoring, requiring new regulatory } \\
\text { constructs }\end{array}$ & $\begin{array}{l}\text { * Principle nodes supporting moves towards more } \\
\text { 'coopetition' at platform level with potential for } \\
\text { rapid two-way transfer of design (clinical) and } \\
\text { manufacturing (commercial) data } \\
\text { * While hitherto sequential in nature, } \\
\text { experimentation around increasing SKUs sees } \\
\text { partners leveraging consortium links } \\
{ }^{*} \text { New design rules emerging linked to archetypes } \\
\text { and segmentation around 'personalised' solutions }\end{array}$ \\
\hline $\begin{array}{l}\text { Simultaneous } \\
\text { pathway } \\
\text { (dPPI and } \\
\text { dSCl) } \\
\text { Cases } \\
9-16\end{array}$ & $\begin{array}{l}\text { * To deliver a diverse scope of applications, } \\
\text { central nodes coordinating 'unique pairings' } \\
\text { for 'platform-based strategies' } \\
\text { * Hybrid role of central nodes based around } \\
\text { evolving modes of dPPI and/or dSCl; often } \\
\text { interchangeable with multiple combinations } \\
\text { in play } \\
\text { * Central nodes increasingly using } \\
\text { language around 'new measures', 'levels of } \\
\text { 'modularisation', 'scale juxtapositioning,' } \\
\text { capabilities for 'convergence' and } \\
\text { 'precision' }\end{array}$ & $\begin{array}{l}\text { * To meet future techno-social sector requirements, } \\
\text { PPI and SCI dimensions become fundamentally } \\
\text { different in simultaneous pathways } \\
\text { * Consortium effect enabling shifts from 'passive' } \\
\text { interactions (single-firm) towards unique conversations } \\
\text { and transactions beyond the 'norm' (platform-based) } \\
\text { * While coopetition on quality, dependability, service } \\
\text { and cost required as 'qualifiers', competition now } \\
\text { shifting to other measures, depending on new business } \\
\text { models }\end{array}$ & $\begin{array}{l}\text { * Simultaneous pathways imply a 'de facto' } \\
\text { platform approach with the emergence of new } \\
\text { design rules (supported by the regulator) } \\
\text { * Increasing focus on 'data organization', the E2E } \\
\text { integration of 'modular' and continuous process } \\
\text { innovations that operate at much lower and } \\
\text { unconventional scales } \\
\text { * Radically new supply network configurations } \\
\text { with potential sea-change requirements in supply } \\
\text { collaboration, site location, capacity, inventory, } \\
\text { and customer engagement being assessed using } \\
\text { digital network design tools }\end{array}$ \\
\hline
\end{tabular}


On-line Appendix A1 . Main research phases, data sources, data collection and analytical tools

\begin{tabular}{|c|c|c|c|c|c|}
\hline $\begin{array}{l}\text { Research } \\
\text { Phase }\end{array}$ & Consortia contact & $\begin{array}{l}\text { Data collection (initial } 2 \text { phases) } \\
2011-2016 \text { (sequential cases); } \\
2014-2016 \text { (sequential and } \\
\text { simultaneous cases) }\end{array}$ & Data analysis & $\begin{array}{l}\text { Data collection (final } \\
\text { phase) and analysis } \\
2016-2018 \text { (sequential and } \\
\text { simultaneous cases) }\end{array}$ & $\begin{array}{c}\text { Final analysis and } \\
\text { synthesis of findings } \\
(2018-2019)\end{array}$ \\
\hline Data sources & $\begin{array}{l}\text { Consortia members; } \\
\text { academic and sector } \\
\text { specialists not } \\
\text { aligned to consortia } \\
\text { under study }\end{array}$ & $\begin{array}{l}\text { Senior industrialists, managers, } \\
\text { engineers, and scientists; } \\
\text { Academic-Industry workshop-type } \\
\text { engagements focused on specific } \\
\text { themes and consortia outcomes; } \\
\text { lab-scale, pilot-scale and production } \\
\text { plant visits }\end{array}$ & $\begin{array}{l}\text { Workshop reports; field } \\
\text { notes; Academic } \\
\text { publications; } \\
\text { White papers developed } \\
\text { by consortia members }\end{array}$ & $\begin{array}{l}\text { Senior industrialists, } \\
\text { managers, engineers, and } \\
\text { scientists; } \\
\text { Demonstrator visits }\end{array}$ & $\begin{array}{l}\text { Senior industrialists, } \\
\text { managers, engineers, } \\
\text { and scientists; Coded } \\
\text { interviews and } \\
\text { workshop-type } \\
\text { engagements. All other } \\
\text { data sources from initial } \\
2 \text { phases and final } \\
\text { phase }\end{array}$ \\
\hline $\begin{array}{l}\text { Data collection } \\
\text { and } \\
\text { instruments }\end{array}$ & $\begin{array}{l}\text { Face-to-face } \\
\text { engagements; } \\
\text { teleconferences; } \\
\text { follow-on research } \\
\text { grant co- } \\
\text { development with } \\
\text { industrial and } \\
\text { industry partners }\end{array}$ & $\begin{array}{l}\text { Industrial system mapping } \\
\text { techniques: 'micro-maps' charting } \\
\text { evolutions and patterns pre-2011 and } \\
\text { from 2011-2016; } \\
\text { Interview protocols; Broad open- } \\
\text { ended questions common to and } \\
\text { across cases; follow up based on } \\
\text { specific and emerging themes during } \\
\text { workshops; } \\
\text { Further evidence to support } \\
\text { (academic papers and other } \\
\text { secondary data relating to consortia } \\
\text { to validate and give context) }\end{array}$ & - & $\begin{array}{l}\text { Industrial system mapping } \\
\text { charting evolutions 2016- } \\
2018 \text {; Interview protocols are } \\
\text { case and respondent } \\
\text { specific; Created dynamically } \\
\text { in this 2-year final phase as } \\
\text { the data collection process } \\
\text { unfolds; To close out phase, } \\
\text { key stakeholders are asked } \\
\text { to comment on emerging } \\
\text { trends and generalised } \\
\text { patterns from initial } 2 \text { phases } \\
\text { of data analysis }\end{array}$ & $\begin{array}{l}\text { Specific questions or } \\
\text { information asked to } \\
\text { some respondents in } \\
\text { order to verify alternative } \\
\text { explanations and } \\
\text { correctness of our } \\
\text { interpretation. }\end{array}$ \\
\hline $\begin{array}{l}\text { Data analysis } \\
\text { tools }\end{array}$ & - & 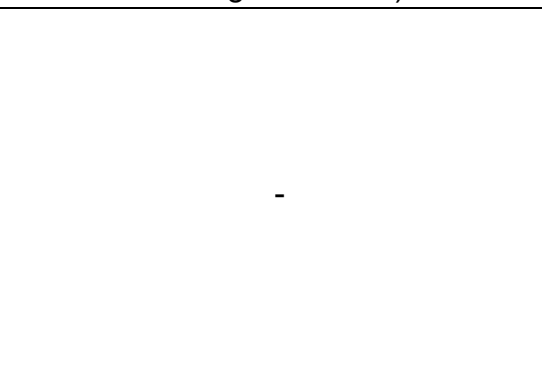 & $\begin{array}{l}\text { Case summary 'dossiers' } \\
\text { capturing value and risk } \\
\text { propositions; network } \\
\text { analysis (NA) and coding } \\
\text { procedures followed to } \\
\text { operationalise the main } \\
\text { constructs, identify } \\
\text { patterns and make } \\
\text { interpretations }\end{array}$ & $\begin{array}{l}\text { NA to highlight patterns } \\
\text { relating to experimentation } \\
\text { and specific sub-networks; } \\
\text { Coding procedures as } \\
\text { before; Construct-oriented } \\
\text { comparisons are made to } \\
\text { highlight patterns }\end{array}$ & $\begin{array}{l}\text { NA to draw inferences } \\
\text { for entire networks and } \\
\text { innovation pathways } \\
\text { using sub-networks; } \\
\text { Construct-oriented } \\
\text { comparisons are made } \\
\text { to highlight patterns and } \\
\text { codify key trends }\end{array}$ \\
\hline
\end{tabular}




\begin{tabular}{|c|c|c|}
\hline & $\begin{array}{l}\text { uential Network Experimentation (cases 1-8) } \\
\text { I or dSCl focus }\end{array}$ & $\begin{array}{l}\text { Simultaneous Network Experimentation (cases 9-16) } \\
\mathrm{dPPI} \text { and } \mathrm{dSCl} \text { focus }\end{array}$ \\
\hline (1) & $\begin{array}{l}\text { Batch development (base case) } \\
\text { dPPI: Improving lab-scale 'design of experiment' (DoE) approaches } \\
\text { Context: Low volume and low variety; API unit operations }\end{array}$ & $\begin{array}{l}\text { (9) Digital workflow } \\
\text { dPPI: Demonstrating feasibility for batch and continuous processes } \\
\text { dSCI: Optimising performance in line with dPPI } \\
\text { Context: E2E Proof-of-Concepts; High volume and low variety }\end{array}$ \\
\hline (2) & $\begin{array}{l}\text { Batch processing with off-line analytics } \\
\text { dPPI: Reducing conventional batch-to-batch variation } \\
\text { Context: High volume and low variety; API unit operations }\end{array}$ & $\begin{array}{l}\text { (10) Plug-and-Play } \\
\text { DPPI: Implementing continuous-dominant processes with analytics } \\
\text { DSCI: Optimising performance in line with dPPI } \\
\text { Context: API; Mobile platforms; High volume and low variety dominant }\end{array}$ \\
\hline (3) & $\begin{array}{l}\text { Batch processing with on-line analytics } \\
\text { dPPI: Reducing conventional batch-to-batch variation; implementing } \\
\text { process analytics } \\
\text { Context: High volume and low variety dominant; API unit operations }\end{array}$ & $\begin{array}{l}\text { (11) Continuous Filtration } \\
\text { dPPI: Implementing continuous-dominant processes with analytics } \\
\text { dSCI: Optimising performance in line with dPPI } \\
\text { Context: Drug product; Agile processes; Low volume and high variety }\end{array}$ \\
\hline (4) & $\begin{array}{l}\text { Hybrid processing systems with on-line analytics } \\
\text { dPPI: Reducing conventional batch-to-batch variation; implementing } \\
\text { process analytics and continuous mode for selected unit operations } \\
\text { Context: High volume and low variety dominant; API unit operations }\end{array}$ & $\begin{array}{l}\text { (12) Next-Generation RFID } \\
\text { dPPI: E2E integration with continuous-dominant processes (API and Drug Product) } \\
\text { dSCI: Optimising performance in line with dPPI } \\
\text { Context: Digital Supply; Intelligent packs; High volume and low variety }\end{array}$ \\
\hline (5) & $\begin{array}{l}\text { Make-to-stock supply (base case) } \\
\text { dSCl: Reducing inventory levels } \\
\text { Context: High volume and low variety; commercial supply }\end{array}$ & $\begin{array}{l}\text { (13) Micro-factories } \\
\text { dPPI: Implementing continuous-dominant processes with analytics } \\
\text { SCI: Optimising performance in line with dPPI } \\
\text { Context: API; Mobile platforms; Low volume and high variety }\end{array}$ \\
\hline (6) & $\begin{array}{l}\text { Printed electronics } \\
\text { dSCl: Optimising inventory levels } \\
\text { Context: High volume and low variety; commercial supply }\end{array}$ & $\begin{array}{l}\text { (14) Continuous Extrusion and 3D-Print } \\
\text { dPPI: Implementing continuous-dominant processes with analytics } \\
\text { dSCl: Optimising performance in line with dPPI } \\
\text { Context: Drug product; Agile processes; Low volume and high variety }\end{array}$ \\
\hline (7) & $\begin{array}{l}\text { Localised on-demand supply } \\
\text { dSCl: Optimising inventory levels } \\
\text { Context: High volume and low variety dominant; clinical supply }\end{array}$ & $\begin{array}{l}\text { (15) Next-Gen product concepts } \\
\text { dPPI: E2E integration with continuous-dominant processes (API and Drug Product) } \\
\text { dSCI: Optimising performance in line with dPPI } \\
\text { Context: Digital supply; Intelligent packs; Low volume and high variety }\end{array}$ \\
\hline (8) & $\begin{array}{l}\text { Adaptive assembly-to-order solutions } \\
\text { dSCl: Optimising inventory levels } \\
\text { Context: High volume and low variety dominant; clinical supply }\end{array}$ & $\begin{array}{l}\text { (16) Digitalisation Lab } \\
\text { dPPI: Demonstrating feasibility of continuous processes } \\
\text { dSCl: Optimising performance in line with dPPI } \\
\text { Context: E2E Proof-of-Concept; Low/high volume and high/low variety }\end{array}$ \\
\hline
\end{tabular}




\section{Online Appendix A3. Network Analysis}

Network \#1. Sequential pathway (dPPI first)

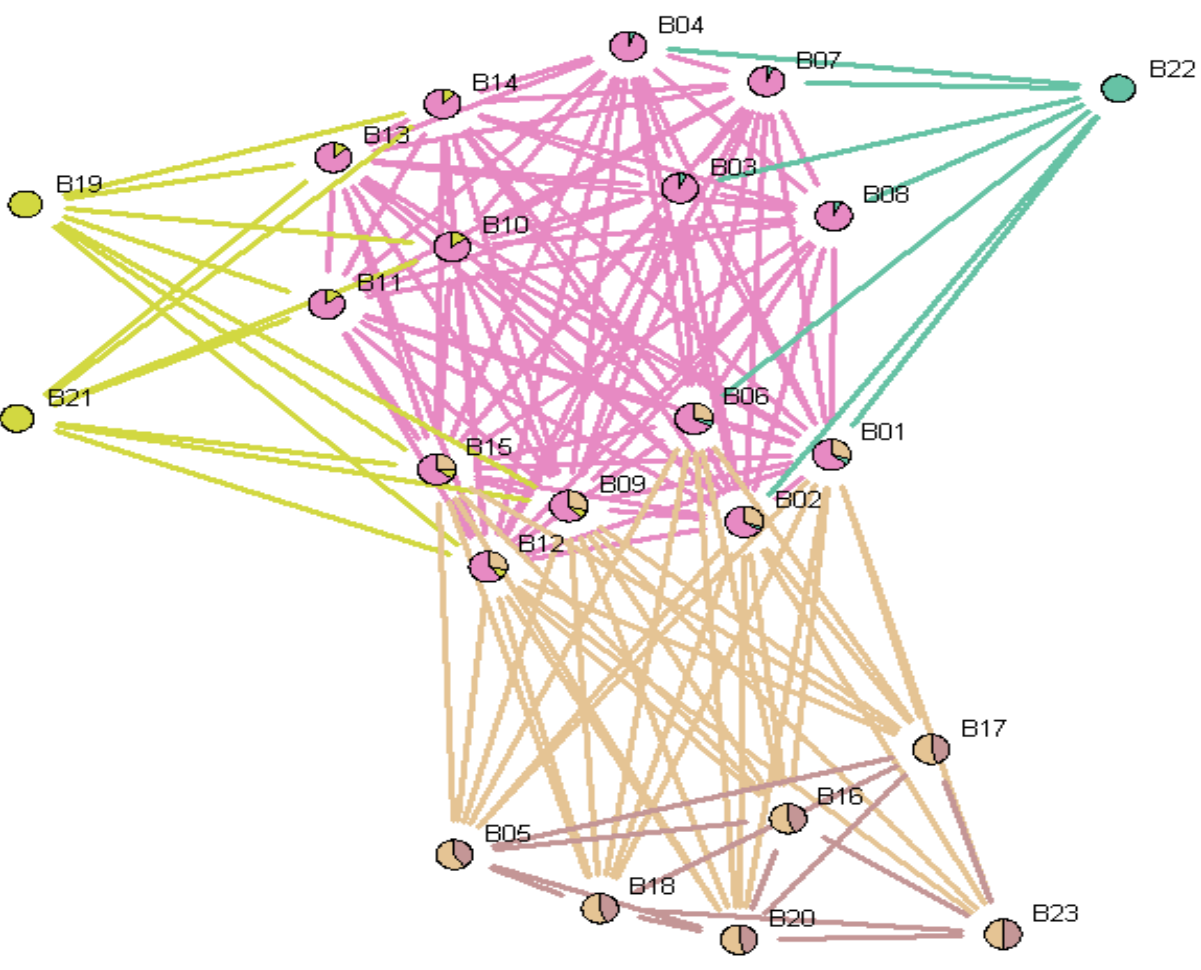

\begin{tabular}{|l|c|c|c|}
$\begin{array}{l}\text { NETWORK \# 1 } \\
\text { Entity }\end{array}$ & ID & Eigenvector & \multicolumn{1}{c}{ Sub-network } \\
\hline Academic Institution 1 & B09 & 1.00 & Integration; Design Rules \\
\hline Academic Institution 2 & B12 & 1.00 & Integration; Design Rules \\
\hline MNC 1 & B01 & 0.97 & Integration; Design Rules \\
\hline MNC 2 & B02 & 0.97 & Integration; Design Rules \\
\hline Academic Institution 3 & B15 & 0.97 & Integration; Design Rules \\
\hline MNC 3 & B06 & 0.95 & Integration; Design Rules \\
\hline Academic Institution 4 & B10 & 0.80 & Application \\
\hline Academic Institution 5 & B11 & 0.80 & Application \\
\hline Academic Institution 6 & B13 & 0.80 & Application \\
\hline Academic Institution 7 & B14 & 0.80 & Application \\
\hline MNC 4 & B03 & 0.78 & Application \\
\hline MNC 5 & B04 & 0.78 & Application \\
\hline MNC 6 & B07 & 0.78 & Application \\
\hline MNC 7 & B08 & 0.78 & Application \\
\hline MNC 8 & B05 & 0.56 & Application \\
\hline SME 1 & B16 & 0.56 & Transition; Design Rules \\
\hline SME 2 & B17 & 0.56 & Transition; Design Rules \\
\hline SME 3 & B18 & 0.56 & Transition; Design Rules \\
\hline AMSCI Funding Body & B20 & 0.56 & Application \\
\hline MMIP & B22 & 0.46 & Application \\
\hline ReMediES consortium & B23 & 0.43 & Application \\
\hline EPSRC Research Council & B19 & 0.43 & Application \\
\hline Knowledge Transfer Network & B21 & 0.43 & Application \\
\hline
\end{tabular}


Network \#2. Sequential pathway (dSCl first)

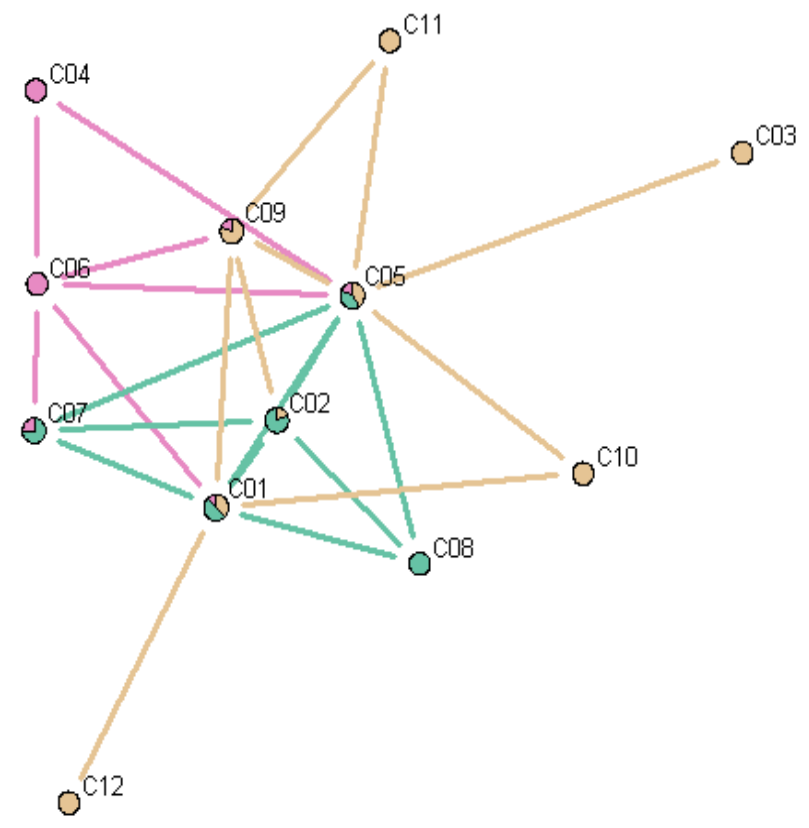

\begin{tabular}{|l|c|c|c|}
$\begin{array}{l}\text { NETWORK \# 2 } \\
\text { Entity }\end{array}$ & ID & Eigenvector & \begin{tabular}{c} 
Sub-network \\
\hline Academic Institution 1
\end{tabular} \\
\hline MNC 1 & 05 & 1.00 & Integration; Design Rules \\
\hline MNC 2 & C01 & 0.89 & Integration; Design Rules \\
\hline UK Regulator & C02 & 0.70 & Integration; Design Rules \\
\hline SME 4 & C09 & 0.68 & Application \\
\hline UK Centre for Process Innovation & C06 & 0.67 & Application \\
\hline SME 5 & C07 & 0.62 & Transition; Design Rules \\
\hline AMSCI Funding Body & C08 & 0.49 & Transition; Design Rules \\
\hline CMAC Consortium & C10 & 0.36 & Application \\
\hline Logistics Provider 1 & C11 & 0.32 & Application \\
\hline Pharmacy Chain 1 & C04 & 0.32 & Application \\
\hline External specialist supplier & C03 & 0.19 & Application \\
\hline
\end{tabular}


Network \#3. Simultaneous pathway

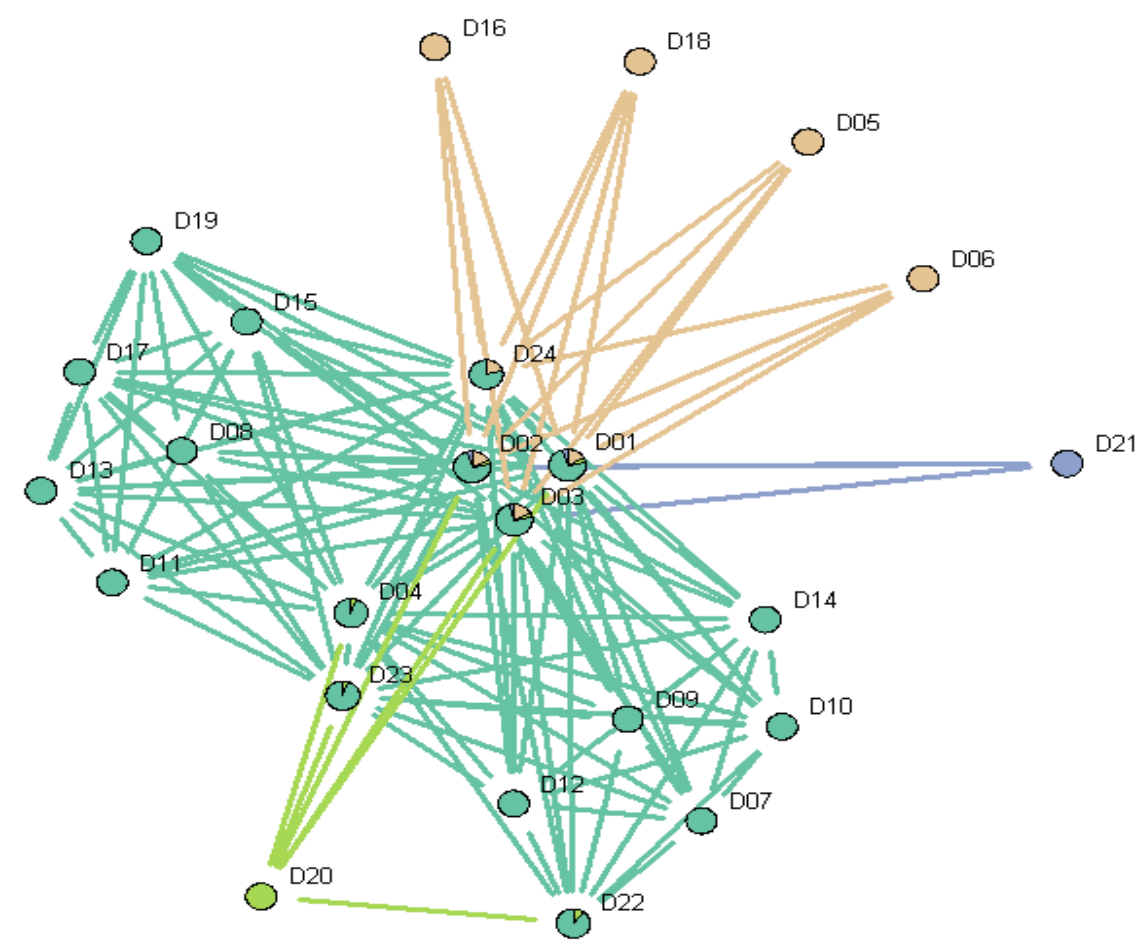

\begin{tabular}{|c|c|c|c|}
\hline \multicolumn{4}{|l|}{ NETWORK \# 3} \\
\hline MNC 1 & D01 & 1.00 & Integration; Design Rules \\
\hline Academic Institution 1 & D03 & 1.00 & Integration; Design Rules \\
\hline MNC 2 & D02 & 0.94 & Integration; Design Rules \\
\hline Academic Institution 2 & D04 & 0.90 & Integration; Design Rules \\
\hline IIM (MNC 1) & D24 & 0.85 & Integration; Design Rules \\
\hline CMAC Consortium & D23 & 0.82 & Integration; Design Rules \\
\hline SME 1 & D07 & 0.64 & Transition; Design Rules \\
\hline SME 6 & D08 & 0.62 & Transition; Design Rules \\
\hline SME 7 & D11 & 0.62 & Transition; Design Rules \\
\hline SME 8 & D13 & 0.62 & Transition; Design Rules \\
\hline SME 9 & D15 & 0.62 & Transition; Design Rules \\
\hline SME 10 & D17 & 0.62 & Transition; Design Rules \\
\hline SME 11 & D09 & 0.62 & Transition; Design Rules \\
\hline SME 12 & D10 & 0.62 & Transition; Design Rules \\
\hline SME 2 & D12 & 0.62 & Transition; Design Rules \\
\hline SME 3 & D14 & 0.62 & Transition; Design Rules \\
\hline SME 13 & D19 & 0.57 & Transition; Design Rules \\
\hline UK Skills Agency & D22 & 0.54 & Transition; Design Rules \\
\hline UK Regulator & D20 & 0.42 & Application \\
\hline SME 4 & D05 & 0.33 & Application \\
\hline SME 14 & D18 & 0.33 & Application \\
\hline SME 15 & D06 & 0.30 & Application \\
\hline UK Centre for Process Innovation & D16 & 0.30 & Application \\
\hline AMSCI Funding Body & D21 & 0.26 & Application \\
\hline
\end{tabular}


Online Appendix A4. Summary of observed outputs and outcomes in sequential and simultaneous pathways

\begin{tabular}{|c|c|c|c|}
\hline \multirow[b]{2}{*}{$\begin{array}{c}\text { Case } \\
\#\end{array}$} & \multicolumn{2}{|c|}{ OUTPUTS } & \multirow{2}{*}{ Comments on the Outcomes Observed in Applications sub-networks } \\
\hline & $\begin{array}{l}\text { Value } \\
\text { Creation }\end{array}$ & Risk & \\
\hline 1 & Conventional & Low & $\begin{array}{l}\text { Base case; Traditional 'Design of experiment' approaches in R\&D driving } 3 \sigma-4 \sigma \text { sector performance; Often significant carry-overs from } \\
\text { development with evidence of temporal trade-offs, as default, at larger scale }\end{array}$ \\
\hline 2 & Conventional & Low & $\begin{array}{l}\text { Incremental improvements to multiple arrangements and repetitions of unit operations in batch-mode; continued spends of c. } 20 \% \text { of } \\
\text { revenues on rework, inspection, and testing }\end{array}$ \\
\hline 3 & Conventional & Low & Incremental improvements to existing processes; emerging QbD principles but quality control regimes still the norm \\
\hline 4 & Novel & High & $\begin{array}{l}\text { Targeting capabilities for better prediction of experimental outcomes; open access across consortia with adoption of Electronic } \\
\text { Laboratory Notebooks for the rapid exchange of reaction and processing data }\end{array}$ \\
\hline 5 & Conventional & Low & $\begin{array}{l}\text { Base case; traditional batch processing models driving high levels of inventory; for the top } 25 \text { pharma companies combined, stock } \\
\text { value is estimated to be in the order of } \$ 100-150 \text { billion }\end{array}$ \\
\hline 6 & Conventional & Low & $\begin{array}{l}\text { Compliance over performance remit; novel value potential (and currently far from realisation) sees opportunities for flexible 'best } \\
\text { before' labels that might disrupt multiple elements of hitherto conventional delivery models }\end{array}$ \\
\hline 7 & Conventional & Low & $\begin{array}{l}\text { Incremental changes to paper batch record system and manually-operated production lines; Potential for control systems and data } \\
\text { management to enable more frequent and lower volume runs than is currently achievable }\end{array}$ \\
\hline 8 & Novel & High & $\begin{array}{l}\text { Better 'targeted' designs through data analytics; Potential to collapse standard transaction and processing times and eliminate costly } \\
\text { write-offs of unused clinical stock, typically in excess of } £ 50 \text { million per MNC per year }\end{array}$ \\
\hline 9 & Conventional & Low & $\begin{array}{l}\text { Incremental changes to-date given the bench-marking performance remit; initial concurrent thinking around new supply network design } \\
\text { principles linked to PPls }\end{array}$ \\
\hline 10 & Novel & Medium & $\begin{array}{l}\text { Recently validated 'targeted' API-specific applications in mobile continuous crystallization processing with first-time translation beyond } \\
\text { proof of concept experimentation and pilots to front-line manufacturing use. }\end{array}$ \\
\hline 11 & Novel & Medium & $\begin{array}{l}\text { Step change to continuous-mode enabled by predictive capabilities; Reduced risk in scale-up of manufacturing processes and } \\
\text { combinations of different unit operations; Opportunities to accelerate the design and modelling of new and more challenging APIs }\end{array}$ \\
\hline 12 & Novel & Medium & $\begin{array}{l}\text { Initial breakthroughs with the development of concepts to replace industry standard rigid 'temptales' with a flexible label based on } \\
\text { Near-Field-Communications (i.e. utilising an Android mobile phone to read the data) }\end{array}$ \\
\hline 13 & Novel & High & $\begin{array}{l}\text { Focus on 'niche' product-process archetypes with lower inventories, shorter times to market, with scale-matching; platform-based } \\
\text { design principles emerging based on new paradigms of 'process intensification', 'miniaturization' and 'combinations' in continuous } \\
\text { modes; Digital twins linked to integrated continuous 'direct-to-dose' schemes }\end{array}$ \\
\hline 14 & Novel & High & $\begin{array}{l}\text { Conventional PPIs in dosage form design and processing cannot meet future needs of personalised medicine. Extrusion technology } \\
\text { demos now producing oral solid dosage forms in one continuous process; With additional 3D-printing functionality, might provide viable } \\
\text { options for extremely flexible dosing, rapid and optimised product design, low material usage and on-demand supply. }\end{array}$ \\
\hline 15 & Novel & High & $\begin{array}{l}\text { New process trials with potential to deliver savings of } £ 2 \text { million on materials and distribution per annum per MNC; 'just-in-time' } \\
\text { technologies demonstrating potential for reducing lead-times from } 4-6 \text { months to }<1 \text { week }\end{array}$ \\
\hline 16 & Novel & High & $\begin{array}{l}\text { High-level strategic goal involves using a range of diverse digital technologies to 'connect' previously disparate upstream and } \\
\text { downstream operations. Key to this is integration of data generated along the product lifecycle in a variety of digital contexts }\end{array}$ \\
\hline
\end{tabular}

